\title{
Analysis of Genetically Determined Gene Expression Suggests Role of Inflammatory Processes in Etiology of Exfoliation Syndrome
}

Jibril Hirbo ( $\square$ jibril.hirbo@vumc.org )

Vanderbilt University School of Medicine https://orcid.org/0000-0002-8932-7311

Francesca Pasutto

Friedrich-Alexander-Universität Erlangen-Nürnberg (FAU) https://orcid.org/0000-0002-0026-5362

Eric Gamazon

Vanderbilt University School of Medicine

Patrick Evans

Vanderbilt University School of Medicine

Priyanka Pawar

Vanderbilt Eye Institute

Daniel Berner

Department of Ophthalmology, Universitätsklinikum Erlangen, Friedrich-Alexander-Universität Erlangen-

Nürnberg

Julia Sealock

Vanderbilt University Medical Center https://orcid.org/0000-0002-9346-3498

Ran Tao

Vanderbilt University School of Medicine

Peter Straub

Vanderbilt University Medical Center

Anuar Konkashbaev

Vanderbilt University School of Medicine

Max Breyer

Vanderbilt University School of Medicine

Ursula Schlötzer-Schrehardt

University Hospital Erlangen-Nuremberg

Andre Reis

Universität Erlangen-Nürnberg https://orcid.org/0000-0002-6301-6363

Milam Brantley

Vanderbilt Eye Institute

Chiea Chuen Khor

Genome Institute of Singapore https://orcid.org/0000-0002-1128-4729 


\section{Karen Joos}

Vanderbilt Eye Institute https://orcid.org/0000-0002-6210-4126

\section{Nancy Cox}

Vanderbilt University Medical Center

\section{Article}

Keywords: Exfoliation syndrome, XFS, Transcriptomic Wide Association Studies, inflammatory processes

Posted Date: August 11th, 2020

DOI: https://doi.org/10.21203/rs.3.rs-53203/v1

License: (c) (i) This work is licensed under a Creative Commons Attribution 4.0 International License. Read Full License 


\section{Analysis of Genetically Determined Gene Expression Suggests Role of Inflammatory Processes in Etiology of Exfoliation Syndrome}

Hirbo, Jibril. ${ }^{1,4}$;* Pasutto, F. ${ }^{2}$; Gamazon, Eric R. ${ }^{1,4,5^{*}}$; Evans, Patrick ${ }^{1}$; Pawar, Priyanka ${ }^{3}$; Berner, Daniel. ${ }^{7}$; Sealock, Julia ${ }^{1}$; Tao, Ran ${ }^{6}$; Straub, Peter S. ${ }^{1}$; Konkashbaev, Anuar I. ${ }^{1}$; Breyer, M. ${ }^{1}$; Schlötzer-Schrehardt, Ursula ${ }^{7}$; Reis, André2; Brantley, Milam A., Jr. ${ }^{3}$; Khor, CC. ${ }^{*}$; Joos, Karen M. ${ }^{3}$; Cox, Nancy J. ${ }^{1,4 *}$

1. Genetic Medicine, Vanderbilt University School of Medicine, Nashville, TN, United States. 2. Institute of Human Genetics, Friedrich-Alexander-Universität Erlangen-Nürnberg FAU, Erlangen, Germany

3. Vanderbilt Eye Institute, Vanderbilt University Medical Center, Nashville, TN, United States.

4. Vanderbilt Genetics Institute, Nashville, TN, United States 37232

5. Clare Hall and MRC Epidemiology Unit, University of Cambridge, Cambridge, United Kingdom

6. Biostatistics, Vanderbilt University School of Medicine, Nashville, TN, United States.

7. Department of Ophthalmology, Universitätsklinikum Erlangen, Friedrich-AlexanderUniversität Erlangen-Nürnberg, Erlangen, Germany

8. Singapore Eye Research Institute

*Corresponding Authors: jibril.hirbo@vumc.org; nancy.j.cox@vumc.org;

ericgamazon@gmail.com; khorcc@gis.a-star.edu.sg 


\begin{abstract}
Exfoliation syndrome (XFS) is an age-related systemic disorder characterized by excessive production and progressive accumulation of abnormal extracellular material, with pathognomonic ocular manifestations. It is the most common cause of secondary glaucoma, resulting in widespread global blindness. We performed Transcriptomic Wide Association Studies using PrediXcan models trained in 48 GTEx tissues to identify genetically-determined gene expression changes associated with XFS risk, leveraging on results from a global GWAS that included 123,457 individuals from 24 countries. Twenty-eight genes in the chr15q22-25 region showed statistically significant associations in both single-tissue and multi-tissue analysis. Reduced models for these genes that excluded variants in LD with the known LOXL1 signal showed genome-wide significant association signals in nine genes, independently of LOXL1. Out of these ten genes, mRNA transcript levels for ARID3B, CD276, LOXL1, NEO1, SCAMP2, and $U B L 7$ were significantly decreased in iris tissues from XFS patients compared to control samples. Genes with genetically determined expression changes in XFS were significantly enriched for genes associated with inflammatory conditions. We further explored the health consequences of high susceptibility to XFS using a large electronic health record and observed a higher incidence of XFS comorbidity with inflammatory and connective tissue diseases. Our results implicate a role for connective tissues and inflammation in the etiology of XFS. Targeting the inflammatory pathway may be a potential therapeutic option to reduce progression in XFS.
\end{abstract}




\section{Introduction}

Exfoliation syndrome (XFS) is an age-related systemic disorder characterized by excessive production and progressive accumulation of abnormal extracellular material, with pathognomonic ocular manifestations. ${ }^{1,2}$ It is the most common cause of secondary glaucoma, resulting in widespread global blindness. ${ }^{3}$ In addition to ocular manifestations, exfoliation syndrome deposits have been observed in visceral organs, such as the lung, kidney, liver and gallbladder. ${ }^{2,4}$ In addition to elastic tissue disorders, XFS has also been associated with increased risk of vascular diseases. ${ }^{5-7}$ Associations of XFS to several systemic biomarkers of inflammation, including complement components and homocysteine, have also been reported..$^{3,8,9}$

Genetic mechanisms have substantial influence on XFS etiology as evidenced in family and twin studies. ${ }^{10,11}$ There have been eight genome-wide association studies (GWAS) of XFS, ${ }^{7,12-18}$ three of which include meta-analysis, ${ }^{7,12,13}$ that have cumulatively identified $>60$ associated genetic variants. The largest meta-analysis of XFS involved $>123,000$ individuals (13,620 XFS cases, 109,837 controls) from 24 countries across six continents and identified seven loci with the strongest association signal in chromosome 15 near the lysyl oxidase-like 1 gene (LOXL1). The signal on chr15 involved 54 potential causal variants. Overall, (i) two missense variants in LOXL1, rs1048661 (encoding LOXL1 p.Leu141Arg) and rs3825942 (p.Gly153Asp), are likely to confer risk of developing XFS, with very high heterogeneity across populations because the alleles show an effect reversal, ${ }^{12,13,16,19-22}$ (ii) the associated variants in the locus showed population-specific frequency and linkage disequilibrium (LD) patterns, ${ }^{12,16,21}$ (iii) haplotypes that carry the risk alleles depending on the population are correlated with reduced LOXL1 expression levels, however, (iv) no clear functional effects for the haplotypes that represent the two variants have been shown. ${ }^{7,13,23,24}$ The non-coding variants associated with 
XFS at this chr15 locus could confer regulatory effects. Some of these non-coding variants regulate expressions of the sentinel LOXL1 and the neighboring STRA6 gene. ${ }^{7,25,26}$

After considering all the reports on genetic architecture of XFS to date, we hypothesize that analysis of the contribution of the genetically-determined component of gene expression to XFS risk can provide a powerful method to elucidate genes involved in XFS. We used a genebased method, PrediXcan ${ }^{27}$, implemented on GWAS summary statistics (Summary PrediXcan; S-PrediXcan $)^{28}$ to identify genetically determined gene expression traits associated with disease risk. Models were trained on 48 GTEx tissues to estimate the correlation between geneticallydetermined gene expression and XFS risk, leveraging on GWAS summary statistics from a previously reported multi-ethnic study. ${ }^{13}$ We also analyzed individual-level GWAS data from four additional European ancestry populations, two German, one Italian ${ }^{7}$ and one American. ${ }^{27}$ We performed extensive statistical analysis, followed by functional validation in human iris tissues of the prioritized top gene-level associations. Finally, to gain clinical insights into our findings, we explored the health consequences to individuals carrying high XFS genetic risk in a large biobank with links to electronic health records. 


\section{$\underline{\text { Results }}$}

PrediXcan Analysis

We performed single-tissue PrediXcan analysis of the global multi-ethnic GWAS $(13,620$ XFS cases and 109,837 controls) summary data, identifying 23 genes (defined as signals with $P<2.02 \times 10^{-7}$ after Bonferroni corrections) on chromosome 15: CYP1A2, CYP1A1, STOML1, LOXL1, ISLR2, RPP25, INSYN, ISLR, STRA6, CD276, NEO1, ARID3B, COX5A, PML, CPLX3, LMAN1L, UBL7, MPI, CLK3, CSK, SEMA7A, TBC1D21, and NPTN (Figure 1a, Suppl. Table S2).

To determine the joint effects of gene expression variation predicted across all 48 tissues analyzed, we performed a multivariate regression multi-tissue analysis. Each of the 23 associations from the single-tissue PrediXcan analysis remained significant in multi-tissue analysis (Suppl. Table S3). Additionally, five genes within the same region that were associated with XFS at subgenome-wide significance in the single-tissue analysis $(\mathrm{p}<3.02 \mathrm{e}-6)$ were associated in the multi-tissue analysis: $A D P G K(\mathrm{p}=7.32 \mathrm{E}-07), C Y P 11 A 1(\mathrm{p}=1.36 \mathrm{E}-16), H E X A$ $(\mathrm{p}=1.03 \mathrm{E}-06)$, PARP6 ( $\mathrm{p}=1.82 \mathrm{E}-06), S C A M P 2(\mathrm{p}=1.65 \mathrm{E}-10)$. All genes mapped to chromosome region 15q22-25, spanning 3 Megabases (Figure 1b).

Seven additional genes located on chromosomes 1 ( LGR6 p=2.20E-06; SDHB p=8.07E08), 6 (PRRT1 p=9.10E-07), 8 (PRSS55 p=4.18E-13), 10 (CDH23 p=1.86E-07; PITRM1 $\mathrm{p}=8.45 \mathrm{E}-12)$ and $19(C A L M 3 \mathrm{p}=2.60 \mathrm{E}-07)$, were significantly associated in the multi-tissues analysis (Figure 1a, Suppl. Table S3). All seven signals mapped to genomic regions harboring GWAS SNP variants showing subgenome-wide significance with XFS risk, except for PRRT1, which corresponds to the AGPAT1 locus ${ }^{13}$ The data indicates that combining information across variants in genes and then across tissue expression improves the power to identify additional XFS-associated loci. 
To ensure that the association observed at the 23 genes from the larger multi-ethnic dataset was not an artefact of population structure, we confirmed the signals in a subset of European ancestry individuals (Materials and Methods). Twelve out of the 23 genes in chr15q22-25 region (ten in the single-tissue analysis and two in the multi-tissue analysis) remained genome-wide significant in this European ancestry analysis, whilst the remaining 11 genes remain nominally associated $(\mathrm{p}<0.05)$ (Suppl. Figure S3, Suppl. Table S4, S5, S6).

\section{Correlated Expression Among Significant Genes}

To determine whether the 23 observed association gene signals were artefacts of LD contamination, we performed extensive additional analyses. We calculated the pair-wise correlation in measured expression among the significant genes, using the reference GTEx panel. We checked the relationship between expression correlation for each of the chr15p22-25 genes with LOXL1 and STOML1 and the PrediXcan associations for the two genes in each tissue. We made two important observations from this analysis. First, there was a significant correlation between the correlation of measured gene expression of the other genes in chr15p22-25 with LOXL1 or STOML1 and the gene-level associations with XFS in most tissues (Suppl. Table S7a, Suppl. Figure S4a). Secondly, there is substantial correlation between STOML1 and LOXL1 ( $\mathrm{r}^{2}$ $=0.67, \mathrm{p}=0.009)($ Suppl. Table S7a, Suppl. Figure S4a). These results indicate that the associations by one of the genes might be due to LD contamination or the presence of shared variants in the prediction models of the two genes (Table 2).

To dissect the potential source of LD contamination in the PrediXcan analysis, we looked into the effect of the two GWAS missense variants implicated in XFS that have mostly been linked to $L O X L 1$ and shown to play regulatory roles $^{23}$, followed by the effect of $L O X L 1$ and 
STOML1 signals on chr15q22-25 region observed associations in each tissue. We also determined the effect of shared variants between prediction models for the genes in the region.

We modified our prediction models by excluding: i) rs3825942 missense variant, ii) rs4886776 intronic variant, which is at near perfect LD (pair-wise, $r^{2}=0.982$ ) with the rs1048661 missense variant, and iii) all the variants in our gene models that were in LD with the two variants $\left(r^{2}>0.1\right)$. The two missense variants had wide ranging effect on the genetically predicted expression of many chr15q22-25 region genes, with the largest effect on LOXL1. The strength of the association signals diminished in six of the nine tissues for which we had the gene's predicted expression. Association signals in three of these tissues fell below genome-wide threshold in the global dataset (Suppl. Table S7b). In addition, association signals for seven additional genes in the region besides STOML1 lost genome-wide significance; CD276 (2 tissues), COX5A, CYP1A1, LMAN1L, MPI, SCAMP2 and TBC1D21.

Interestingly, association signals for eight genes were strengthened, four of which attained genome-wide significance threshold in reduced models: INSYN1, CYPIAI, NPTN and LOXL1 (Suppl. Table S7b). These shifts in association strength, i.e., an increase in effect size, seem to be due to the exclusion of select variants (Suppl. Tables S7c, S7d). Moreover, the shifts in association strength are correlated with the excluded variants' level of LD with the missense variant rs3825942 $\left(r^{2}=0.64\right)($ Suppl. Tables S7c, S7d). Notably, the three GWAS variants identified to have effect reversal in South Africans relative to other populations were in high LD with rs3825942 (Suppl. Table S7g). ${ }^{29}$ Our results indicate that the missense variants have enhancing or diminishing effects on the PrediXcan association signals, in chr15q22-25, with XFS, consistent with allele reversal reported for the GWAS variants. ${ }^{13}$ 
To check whether the association signals in the chr15q22-25 region for each tissue were independent of the 'sentinel' LOXL1 signal, we excluded, from the prediction models, variants that were in LD $\left(r^{2}>0.1\right)$ with any variants in LOXL1 or STOML1 tissue models. We also excluded variants that were shared between two or more genes in their original prediction models. Seven of the genes that were associated with XFS at genome-wide threshold in their original models showed diminished signals, including four below significance levels: $U B L 7$, ISLR, LMAN1L and COX5A in reduced models (Suppl. Table S7e). Association signals for CYP1A1 and CYP1A2 were slightly diminished in reduced models relative to the original models, but remained at significant genome-wide thresholds (Suppl. Table S7e). However, association signals for six genes strengthened, four of which attained genome-wide association significance levels in the reduced models: INSYN1, CLK3, CYP1A1 and NEO1 (Suppl. Table S7e). These shifts in association strength seem to be due to few variants that are either in LD with variants in LOXL1 and STOML1 models or are shared with other genes' models (Suppl. Tables S7e, S7f). However, these variants causing the shifts in association signals upon exclusion from the models, were not in LD with the missense rs3825942 variant (Suppl. Table S7g). This indicates that there are signals of allele reversal independent of the known missense variants in LOXL1.

Excluding variants that are in LD with SNPs in the LOXL1/STOML1 models did not have any effect on the association signals for six genes that were associated with XFS at genome-wide threshold in the original models: CSK, STRA6, CD276, ARID3B, MPI \& TBC1D21, with the latter three in testis, for which we had no models for both LOXL1 and STOML1 (Suppl. Table S7e). The results indicate that some of the observed signals were artefacts of LD contamination from LOXL1 and STOML1 (ISLR, LMAN1L and COX5A), while some of the signals were 
masked in the original models (INSYN1, CLK3, CYP1A1 and NEO1). There was inconsistent result for $U B L 7$, where there was no effect in its association signal in a tissue, enhanced effect in another tissue, and diminished signal in two other tissues, one of which went below the genomewide threshold, albeit the reduced model had only a single variant in the prediction (Suppl. Table S7e).

\section{Conditional Analysis}

Conditional analysis was performed in tissues with any genome-wide significant chr15p22-25 region gene signals against the predicted gene expression for the strongest observed signals in the European subset. As in the global dataset, the strongest signals in the European dataset were at LOXL1 (Table 1, Suppl. Figure S3). In all nine of the 48 tissues with LOXL1 predicted expression, only the STOML1 gene showed a significant association signal (in addition to LOXL1) (Table 1, Suppl. Figure S3). After conditioning on LOXL1 in these tissues, the STOML1 signal disappeared, but association signals at SCAMP2 and INSYN1 were observed in artery-aorta and lung tissues, respectively (Table 2, Figure 2a). This indicated that the association of STOML1 with XFS is an artefact of a strong LOXL1 signal, consistent with LOXL1 being the true signal and STOML1 a proxy signal. In addition, association signals for SCAMP2 and INSYN1 were masked by the LOXL1 signal.

In 17 tissues with STOML1 predicted gene expression, we observed significant association signals for 8 other genes (in addition to STOML1) (Table 2, Figure 2b). After conditioning on STOML1 predicted gene expression, associations with four genes (CYP1A1, INSYN, LOXL1, SCAMP2) remained, while association with four other genes (ISLR, LMANIL, $M P I \& S E M A 7 A)$ disappeared. In addition, associations with five more genes (ARID3B, CPLX3, CYP1A2, PML \& UBL7) attained genome-wide significance after the conditional analysis. 
Overall, conditional PrediXcan analysis of genetic signals in the chromosome 15 region in the European dataset in a limited number of tissues was mostly consistent with PrediXcan analysis using the reduced models above. The analysis confirms the associations for $L O X L 1$, ARID3B, CPLX3, CYP1A1, CYPIA2, INSYN1, NEO1, PML, SCAMP2, and UBL7, all of which, except for INSYNI, have been shown to be highly expressed in eye tissues ${ }^{30}$ (Suppl. Figure S5). However, INSYN1 has enhanced expression in brain tissues. ${ }^{31,32}$ Collectively, these results suggest that some of the identified gene-level association signals between XFS and genetically imputed expression were driven by correlation to the strong LOXL1 and its "proxy" STOML1 signal.

\section{Enrichment and pathway analysis}

Genes at genome-wide significance $(\mathrm{p}<2.02 \mathrm{e}-7)$ and nominal significance $(\mathrm{p}<0.05)$ were evaluated for enrichment of known pathways, using Enrichr. ${ }^{33,34}$ Genes at genome-wide significance were enriched for genes reported for, or mapped to, GWAS variants implicated in several caffeine-related (coffee and caffeine consumption, and caffeine metabolism ${ }^{35-37}$ ) and blood pressure ${ }^{38}$ traits. The enrichment for coffee consumption is replicated for the larger gene set that is associated with XFS at nominal significance. ${ }^{39}$ Some of these genes, CYP1Al and CYP $1 A 2,{ }^{40}$ are involved in fatty acid oxidation and estrogen receptor pathways. In addition, these two genes are also observed in the Reactome enrichment of protectin synthesis (Table 3, Suppl.

\section{Table S8).}

Our gene set is also enriched for genes associated with carcinoma and three inflammatory conditions: rheumatoid arthritis, Type 1 diabetes, vitiligo in Jensen Diseases, a database that integrates evidence on disease-gene associations from automatic text mining, manually curated literature, cancer mutation data, and GWAS (https://diseases.jensenlab.org/). 
We further analyzed our gene list against compounds in Drug Signatures Database (DSigDB, http://tanlab.ucdenver.edu/DSigDB), a gene set resource that relates drugs/compounds and their target genes. Our gene set is enriched for genes that are targets of cyclosporin A $(\mathrm{p}=9.66 \mathrm{E}-11)$, and genes that are targets for compounds that are either 1) carcinogenic: Aflatoxin B1, potassium chromate, methyl methanesulfonate and copper sulfate, 2) neuroactive: valproic acid and methamphetamine, 3) neuroprotective: quercetin and epigallocatechin gallate, or 4) analgesic: acetaminophen (Table 3, Suppl. Table S8). Cyclosporin A is an immunosuppressant taken to treat rheumatoid arthritis and other autoimmune conditions, while quercetin and acetaminophen have been shown to have anti-inflammatory effects. ${ }^{41,42}$

Analysis in Gene Set Enrichment Analysis (GSEA) using a ranked association gene list based on effect sizes confirmed some of the enrichment observations using Enrichr. GSEA besides replicating enrichment for acetaminophen, showed enrichment for: 1) six synthetic estrogens, 2) estrogen regulators (Clomifene), 3) antiarrhythmic (quinidine), and 4) an antifungal (ketaconazole) (Table 3, Suppl. Table S8). The gene set was also enriched for genes that were associated with the collagen fibril crosslinking $(F D R=0.0313)$ Reactome pathway. Analysis of the gene list in relation to the latest Reactome library (https://reactome.org/) returned significant enrichment for the endosomal-vacuolar pathway $(\mathrm{p}=8.14 \mathrm{E}-11)$, an enrichment that was replicated in gene sets that were predicted to be downregulated $(\mathrm{p}=3.24 \mathrm{E}-8)$. Our results broadly recapitulated results above, even after excluding genes in HLA and chr17 inversion regions from the enrichment analysis of the gene set $(\mathrm{p}<0.05)$ (Table 3, Suppl. Table S8) 


\section{Quantitative Expression Validation Analysis}

Expression levels of ARID3B, CD276, INSYN1, LOXL1, NEO1, SCAMP2, STOML1 and UBL7 were measured in XFS and control eye tissues. All transcript levels were found to be decreased in iris tissues obtained from XFS patients compared to control samples, with significant differences for ARID3B, CD276, LOXL1, NEO1, SCAMP2 and UBL7 $(p<0.05)$

(Figure 3). INSYNI and STOMLI were not significantly downregulated in diseased eyes relative to normal eyes in validation analysis. STOML1 is the closest gene to and potentially proxy for LOXL1 among those that show association in our PrediXcan results within the chr15q22-25 region. We included it as a negative control in the validation analysis, while $L O X L 1$ was a positive control considering that it had already been shown to exhibit pattern of downregulation in gene expression in diseased relative to normal tissues. ${ }^{13} \mathrm{CD} 276$ was selected for functional validation in eye tissue despite no significant association with XFS in single-tissue analysis in the European ancestry data because it was significantly associated with XFS in multi-tissue analysis in European data. In addition, it was one of the gene association signals which were not affected by excluding variants that were in LD with LOXL1/STOML1 model SNPs in the multiethnic global dataset. Overall, our validation results replicate the associations found using the genetically determined gene expression.

\section{Comorbidity/pleiotropy analysis}

To gain further biological insights into the gene associations we observed in our PrediXcan analysis, we performed logistic regression analysis of both XFS ICD9/10 diagnosis and Polygenic Risk Score generated from the multi-ethnic summary data across the BioVU individuals, Vanderbilt University's electronic health records database linked to genetic 
information, as the target dataset (Materials and Methods). XFS diagnosis was associated with an increased risk of 96 phenotypes in BioVU, including 12 musculoskeletal phenotypes, 4 infectious diseases, and 1 cardiovascular phenotype. These results are consistent with higher comorbidity of diseases affecting inflammation, connective tissue, and the circulatory system in individuals with XFS (Suppl. Table S9a, Suppl. Figure S6a).

XFS polygenic score was not significantly associated with any phenotypes in the analysis (Suppl. Figure S6b). This potentially indicated that PRS generated from the global multi-ethnic GWAS summary might not be powered to detect association with traits in the EHR, and we might require scores from a more homogeneous and a much larger sample size. However, among the top PRS associations, we found several inflammatory diseases (Suppl. Table S9b), consistent with the enrichment results reported above. 


\section{$\underline{\text { Discussion }}$}

We performed gene-based association analysis using GWAS summary statistics and conducted extensive experimental validation of genes associated with XFS. From our PrediXcan analysis, we identified 35 associated genes with XFS, 23 in single-tissue analysis and the rest in multi-tissue analysis. To eliminate the possibility of false-positive results due to LD contamination, we performed extensive additional analyses. First, we performed PrediXcan analysis in reduced models removing variants in LD with the two LOXL1 missense variants associated with XFS, and variants in LOXL1/STOML1 models in both global multiethnic and a subset of European ancestry individuals. Secondly, we conducted conditional analysis of the significant signals in European ancestry individuals. Thirdly, we then filtered signals based on correlated gene expression, LD and shared eQTLs and confirmed thirteen genes to be associated with XFS. Finally, expression analysis in human iris tissues further confirmed six of these seven signals, which were significantly downregulated in diseased XFS relative to normal eye tissues; ARID3B, CD276, LOXL1, NEO1, SCAMP2 and UBL7.

Our results suggest potentially substantial roles of inflammation and environment in the etiology of XFS. All of the six genes prioritized here by prediction and extensive validation analyses have inflammatory roles. ARID3B, CD276, LOXL1 and NEO1 are immunoregulatory molecules involved in the interaction between different tumors and the immune system. ${ }^{43-46}$ SCAMP2 is important in granule exocytosis, a process crucial in membrane fusion in normal cellular functions in diverse systems including the immune system's inflammatory response. ${ }^{47-49}$ CD276 is involved in regulation of Ag-specific T cell-mediated immune responses and participates in the innate immunity-associated inflammatory response. ${ }^{50,51}$ LOXL1 has also been implicated in fibrosis in response to inflammation in human breast cancer, ${ }^{52}$ in liver and lungs in 
model animals. ${ }^{53-55}$ UBL7 encodes a member of the ubiquitin protein family, that is crucial in immune response and regulation of inflammatory response. ${ }^{56-58}$

Genes that show significant association of predicted expression with XFS at nominal significance are enriched for genes associated with three inflammatory conditions: rheumatoid arthritis, Type 1 diabetes and vitiligo in the Jensen Diseases database, with genes associated with the former two conditions enriched even with HLA region excluded. This is also consistent with the enrichment we find in DSigDB and DrugBank for cyclosporin A, acetaminophen and quercetin, which are compounds that have anti-inflammatory effects. ${ }^{59}$

Enrichment of predicted genes in this study in the polyunsaturated fatty acid (PUFA) and steroid derivatives: protectin (Reactome), omega fatty acid and estrogen (WikiPathways) are also consistent with the potential role of inflammation in XFS. Protectin, a derivative of PUFA including Omega-3 that are major components of fish oil, has an anti-inflammatory, antiamyloidogenic, and anti-apoptotic activities in human neural cells. ${ }^{60-62}$ Omega fatty acid has been suggested as an IOP reducing supplements ${ }^{63-65}$ because of its anti-inflammatory effects. ${ }^{66}$ Association of steroid derivative, estrogen with glaucoma has been previously explored with higher levels of estrogen in reduction in IOP and conferring a possible reduced risk of glaucoma. ${ }^{67}$ The synthetic form of estrogen, estradiol, has been shown in a rat glaucoma model to inhibit optic nerve axonal degeneration by inducing a protein that is crucial in protecting RGC from oxidative damage. ${ }^{68,69}$

The association with inflammation is consistent with studies in limited numbers of XFS patients that found elevated inflammatory markers relative to controls, including cytokines, and markers such as interleukin-6 $(I L-6)$ and $I L-8,{ }^{70,71}$ tumor necrosis factor- $\alpha(T N F-\alpha)$ and $Y K L$ $40 .^{72,73}$ However, there are conflicting results for high sensitivity C-reactive protein. ${ }^{74,75}$ 
In addition, the XFS gene sets are enriched for genes that map to variants implicated in coffee and caffeine intake. Effects of caffeine consumption in the etiology of XFS have been studied, on the premise that coffee consumption increases plasma homocysteine levels that are speculated to enhance XFS material formation by contributing to vascular damage, oxidative stress, and extracellular matrix alterations. ${ }^{76-79}$ Consumption of coffee has been reported to have both pro- and anti-inflammatory effects ${ }^{80}$ However, review of fifteen studies on the effect of coffee and caffeine on inflammation inferred the former had anti-inflammatory action, while the latter had complex effects on the inflammatory response with both proinflammatory and antiinflammatory responses reported. ${ }^{81}$ Caffeine might have a neuroprotective role by regulating pathways that produce inflammatory molecules via adenosine receptors in brain cells. ${ }^{82,83}$ Posttranscriptional regulation of LOXL1 gene expression has been also shown to be modulated by caffeine. ${ }^{84}$

Globally, our results of the six novel functionally validated genes also confirm the role of connective tissue involvement in the etiology of XFS. Aung, et al., ${ }^{13}$ demonstrated the role of haplotypes that carry LOXL1 XFS causal coding variants in upregulating extracellular matrix components such as elastin and fibrillin, and increasing cell-cell adhesion. In addition, two of the novel genes in our study, $A R I D 3 B$ and NEO1, among the other six genes identified and validated in both studies, have adhesive roles in the body. ARID3B in conjunction with FDZ5 protein increases adhesion to ECM components, collagen IV, fibronectin and vitronectin, that are components of exfoliation deposits. ${ }^{85,86} \mathrm{NEO} 1$ has also been shown to play adhesive role during organogenesis. ${ }^{87}$

Results from our enrichment analysis of genes associated with XFS are also consistent with a role of dysregulation in connective tissue metabolism in the etiology of XFS. 
Cylosporin_A regulate lysyl oxidase expression and collagen metabolism probably by inhibiting an isomerase involved in protein folding ${ }^{88-90}$ Other anti-inflammatory compounds identified from our enrichment analysis in the current study, epigallocatechin gallate, valproic acid, quercetin, ketoconazole and acetaminophen have also been shown to suppress collagen and/or are anti-fibrotic in variety of tissues by yet to be elucidated mechanism. ${ }^{91-95}$ Moreover, coffee and caffeine inhibit collagen expression and deposition, and have anti-fibrotic effects by blocking expressions and/or by modulating effects of profibrotic factors. ${ }^{96-99}$

Our results that show enrichment in crosslinking of collagen fibrils, a crucial constituent of connective tissues, and endosomal-vacuolar Reactome pathways, in our associated genes further confirm the importance of connective tissues in the etiology of XFS. In addition, there may be anomalies in an endosomal-vacuolar pathway shown to be involved in the accumulation of other aberrant proteins, including: $\mathrm{A} \beta$ peptides, ${ }^{100}$ prion $^{101}$, and Huntingtin ${ }^{102}$ in neurons, and implicated in neurodegeneration. Moreover, inflammation has also been suggested in migratory failure and subsequent deposition of aberrant proteinaceous materials in affected tissues in conjunction with other molecular actors. ${ }^{71,103-108}$

Finally, our comorbidity analysis in the BioVU EHR indicated XFS association with several chronic inflammatory dermatological, musculo-skeletal, respiratory and infectious conditions. Moreover, extracellular matrix dysregulation is also suggested by our PheWAS results indicating XFS comorbidity with Vitamin D deficiency. Vitamin D regulate collagen cross-linking in vitro by upregulating gene expression of specific lysyl hydrogenase and oxidase enzymes. ${ }^{109}$ 


\section{Limitations of the study}

This study has two main limitations. First, despite the fact that GTEx data for the 48 tissues represent the most comprehensive eQTL data set of human tissues, it does not constitute a complete representation of all human tissues and may fail to identify the real causal genes in the unsampled ocular tissue. However, we have confirmed from an ocular tissue database that novel signals identified in this study are robustly expressed in XFS relevant eye tissues. Moreover, recent analysis shows that the majority of the human body tissues exhibit higher degrees of tissue similarities. ${ }^{110}$ In addition, it has been shown that most complex conditions, including XFS, might actually manifest in many diverse tissues in the body. ${ }^{110}$

Second, only a third of the signals identified in the larger data were robustly confirmed in a European dataset at genome-wide significance. This raised the possibility that most of the initial signals identified an artefact of local LD leakage or shared eQTLs with the sentinel LOXL1/STOML1 signal. Using statistical validation with reduced models including no SNPs in LD with sentinel variants, we confirmed associations independent of LOXL1 for at least ten genes including seven that were experimentally validated. In addition, results from a recent study are consistent with two other association gene signals confirmed using multi-tissue analysis of European dataset and PrediXcan of reduced models in multi-ethnic global data, ISLR2 and STRA6. ${ }^{25}$ ISLR2 and STRA6 are both significantly downregulated in tissues of XFS patients together with other key components of the STRA6 receptor-driven Retinoic acid (RA) signaling pathway, and that siRNA-mediated downregulation of RA signaling induces upregulation of LOXL1 and XFS-associated matrix genes in XFS-relevant cell types. ${ }^{25}$ These data indicate that dysregulation of STRA6 and impaired retinoid metabolism are involved in the pathophysiology of XFS syndrome. Retinoic acid, the active metabolite involved in the signaling pathway 
implicated by Berner $e t a l^{25}$ in XFS through regulation of ISLR2, STRA6 and LOXL1, has been shown to control critical checkpoints in inflammation and to promote an inflammatory environment. ${ }^{111-113}$

In summary, our analysis of predicted gene expression and extensive functional analysis in eye tissue prioritized six genes in association with XFS. Our results further confirmed the role of connective tissues and highlighted the importance of inflammation in the etiology of XFS. Thus, molecular elements that underlie the interaction of connective tissue biosynthesis and inflammatory pathways may play a central role in the etiology of XFS. 


\section{Materials and Methods}

We used an extension of PrediXcan ${ }^{28}$ that uses GWAS summary statistics, SPrediXcan, ${ }^{11}$ to analyze GWAS summary statistical data from a multi-ethnic GWAS study on XFS. ${ }^{13}$ This dataset consisted of 13,620 XFS cases and 109,837 controls. We also performed PrediXcan on individual-level genetic data from two independent datasets comprising 4127 cases and 9075 controls. The first dataset comprised case and control samples from three cohorts of European ancestry (two from Germany and one from Italy). The second dataset comprised adult patients of European ancestry at Vanderbilt University Medical Center (VUMC) from the local communities surrounding Nashville, TN. The BioVU cases and controls were genotyped on five different Illumina genotyping arrays; Human660W-Quad, HumanOmni1-Quad, Infinium Omni5-4, OmniExpress-8v1-2-B and Infinium Multi-Ethnic Global-8 (MEGA). The data was processed using established GWAS quality control procedures ${ }^{8}$, and imputed on the Michigan Imputation server. Details on how subject selection for BioVU data and genotyping was performed is found in extended materials and methods section (Supplementary Information).

\section{Statistical Analysis}

We used the gene-based method, PrediXcan, that provides a framework for correlating imputed gene expression with phenotype. ${ }^{9}$ Gene expression prediction models for 48 different human tissues were trained using GTEx v7 data, subsampled to use only the European ancestry samples. Models with non-zero weights that met a set significance criterion $(r>0.10, q<0.05)$ were retained. ${ }^{27}$ Given the lack of eye tissue in the GTEx data, we performed PrediXcan analysis in all available tissues to leverage the shared regulatory architecture of gene expression across tissues. ${ }^{114}$ We referred to the association analysis in each tissue between predicted expression and XFS as "single-tissue analysis." Because XFS is considered a systemic disorder, we also 
aggregated evidence across the different tissues to improve our ability to prioritize genes relative to a single unrelated tissue. We determined the joint effects of gene expression variation predicted across all 48 tissues using the Multi-Tissue PrediXcan (MultiXcan), a multivariate regression method that integrates evidence across multiple tissues taking into account the correlation between the tissues. ${ }^{115,116}$ We refer to this association analysis as "multi-tissue analysis."

We used S-PrediXcan ${ }^{28}$ to analyze all GWAS summary statistic data from the multiethnic study of Aung, et al. ${ }^{13}$. Since the summary-based method has been shown to be conservative and tends to underestimate significance in cases where there is some linkage disequilibrium-structure mismatch between reference and study cohorts, ${ }^{28}$ we retained and reported S-PrediXcan results that had a univariate S-PrediXcan $P<0.0001$. We used Bonferroni adjustment for multiple hypothesis testing. Genome-wide significance for a gene-level association in single-tissue and multi-tissue PrediXcan analysis were defined as $\mathrm{p}<2.02 \mathrm{e}-7$ and $\mathrm{p}<3.02 \mathrm{e}-6$, respectively.

\section{Conditional Analysis and Linkage Disequilibrium Evaluation}

To determine whether multiple association signals within the same locus are due to independent causal genes or statistical artefacts of correlation in measured expression and predicted gene expression for adjacent genes, ${ }^{117}$ we examined the correlation in the gene expression among genome-wide significant genes in the reference GTEx data. We assumed that there is concordance in correlation in measured and predicted gene expressions, but depending on the quality of our predictions, correlation in predicted expression for a pair of genes may be 
missed. We verified the extent of LD in the 1000 genomes database $\mathrm{e}^{118,119}$ between variants in the prediction models for significantly associated genes in each tissue.

To measure potential regulatory effects of the two classical LOXL1 missense variants in our PrediXcan analysis, we excluded them and all the variants in our gene models that were in LD with them (defined as pairwise $r^{2}>0.1$ ) to generate "reduced models". We predicted gene expression and performed association analysis using reduced models in both the global multiethnic and the European subset for the genes in chromosome 15 region. To assess whether the association signals in the chr15q22-25 region for each tissue are independent of the 'classical' LOXL1 signal, we excluded variants in the prediction models of genes in the region that were in LD (pairwise $\mathrm{r}^{2}>0.1$ ) with any variant in the LOXL1 model. In tissues without a LOXL1 model (i.e., $\mathrm{r}^{2}>0.10, \mathrm{q}<0.05$ ), we excluded variants for chr15q22-25 region genes that were in LD (r2>0.1) with variants in the STOML1 models. In addition, we excluded variants that were shared between prediction models for genes in the region. In each case, we performed association analysis using the reduced models and compared the results with the original models.

To determine whether additional genes within the region were significantly associated with XFS, independently of the most highly associated genes (LOXL1 and STOML1) identified in the primary analysis, we performed conditional analysis using the actual individual-level genotype data that included our BioVU cohort and a subset of Aung, et al. consisting of three European ancestry cohorts. For each tissue with a significant association, the conditional analysis was performed on the gene that was the most statistically significant as identified from the initial PrediXcan analysis. We generated genetically determined expression for each individual in the dataset and then performed association analysis using Genetic Association Analysis Under Complex Survey Sampling (SUGEN: version 8.8$)^{120}$ on the individual imputed gene expression 
data, including age, sex, first 5 principal components and relatedness in the regression model. A new logistic regression model was then fit to the case-control data by sequentially adjusting for the expression data of the top significant signals as a covariate. We then performed a metaanalysis for the PrediXcan summary statistics from the four datasets. We repeated this procedure until no genes in the region attained our threshold for statistical significance in the tissues tested ( $<0.05 /$ total \# of e-genes $\mathrm{x} \#$ of tissues tested for each top round of tests).

\section{Enrichment and pathway analysis}

Genes that were predicted to be associated with XFS at genome-wide significance in both single-tissue and multi-tissue analysis, and at nominal significance $(\mathrm{p}<0.05)$ in single-tissue analysis were checked for enrichment of particular categories in several databases using the webbased enrichment tools, Enrichr. ${ }^{33,34}$ This was done by using the strongest signal at nominal significance across the 48 tissues for each of the genes analyzed in PrediXcan. Enrichr implements Fisher's exact test and uses over 100 gene set libraries to compute enrichment. ${ }^{33} \mathrm{We}$ also performed rank-based Gene Set Enrichment Analysis (GSEA) using another web-based enrichment tool, 2019 Webgestalt ${ }^{121-124}$ with a more recent database (Gene Ontology January 2019, KEGG Release 88.2, Reactome ver.66 September 2018 and PANTHER v3.6.1 Jan 2018) and the current Reactome database ver. 69 (June 12 2019). ${ }^{125}$ In this case the strongest signal in the PrediXcan result across the 48 tissues for each of the genes analyzed was used. Based on previous studies indicating limitation in accurately quantifying expression effects of variants in highly polymorphic regions, ${ }^{126,127}$ we also performed enrichment analysis after excluding a total of 310 genes in $\sim 6 \mathrm{Mb}$ chromosomes $6 \mathrm{HLA}$ region (hg19 28Mb-34Mb) that encompassed GPX6 - CUTA genes (238 genes) and $\sim 2.5 \mathrm{Mb}$ chromosome 17 region that encompassed 
CCDC43 - NPPEPS that include the $900 \mathrm{~kb}$ inversion common in population of European ancestries (72 genes).

\section{Quantitative Expression Validation Analysis}

\section{$\underline{\text { Human tissues }}$}

Human donor eyes used for corneal transplantation with appropriate research consent were obtained from donors of European ancestry. Eyes were processed within 20 hours after death. Informed consent to tissue donation was obtained from the donors or their relatives. The protocol of the study was approved by the Ethics Committee of the Medical Faculty of the Friedrich-Alexander-Universität Erlangen-Nürnberg (No. 4218-CH) and adhered to the tenets of the Declaration of Helsinki for experiments involving human tissues and samples.

For RNA and DNA extractions, 12 donor eyes with manifest XFS syndrome (mean age, $77 \pm 9$ years) and 19 normal-appearing control eyes without any known ocular disease (mean age, $74 \pm 6$ years) were used. All individuals who donated the XFS tissues were previously confirmed XFS patients through routine ophthalmologic examination after pupillary dilation. The presence of characteristic XFS material deposits in manifest disease was assessed by macroscopic inspection of anterior segment structures and confirmed by electron microscopic analysis of small tissue sectors. Iris tissues were prepared under a dissecting microscope and rapidly frozen in liquid nitrogen.

\section{$\underline{\text { Real-time PCR }}$}

For quantitative real-time PCR, iris tissues were extracted using the Precellys 24 homogenizer and lysing kit (Bertin, Montigny-le-Bretonneux, France) together with the AllPrep DNA/RNA kit (Qiagen, Hilden, Germany) according to the manufacturer's instructions 
including an on-column DNaseI digestion step using the RNase-free DNase Set (Qiagen). Firststrand cDNA synthesis and PCR reaction was performed as previously described. ${ }^{128}$ Exonspanning primers (Eurofins Genomics, Ebersberg, Germany), designed with Primer 3 software (http://bioinfo.ut.ee/primer3/), are summarized in Suppl. Table S1. Quantitative real-time PCR was performed using the CFX Connect thermal cycler and software (Bio-Rad Laboratories, München, Germany). Probes were run in parallel and analysed with the $\Delta \Delta \mathrm{Ct}$ method. Averaged data represent at least three biological replicates. Unique binding was determined with UCSC BLAST search (https://genome.ucsc.edu/) and amplification specificity was checked using melt curve, agarose gel and sequence analyses with the Prism 3100 DNA-sequencer (Applied Biosystems, Foster City, CA). For normalization of gene expression levels, mRNA ratios relative to the house-keeping gene GAPDH were calculated.

Group comparisons were performed using a Mann-Whitney U test using SPSS v.20 software (IBM, Ehningen, Germany). $P<0.05$ was considered statistically significant.

\section{Testing for Comorbidity/Pleiotropy}

To determine the comprehensive health consequences of high genetic risk to XFS, we performed a phenome-wide association study (PheWAS). ${ }^{129}$ First, we examined the comorbidity of other phecodes with XFS (365.5 - ICD9 365.52/ICD10 H40.14xx) in a total of 752,024 individuals in the VUMC EHR (418,371 females and 333,653 males), by performing logistic regression analysis conditioned on gender, age and the self-reported ancestry as covariates in the regression model. For this analysis we used a total of 600,107 European, 103,209 African, 12,411 Asian and 36,297 other ancestry patients, of which 222 were uncurated XFS cases (coded as 1) and the rest controls. To determine other health consequences of high genetic risk to XFS, 
we performed a PheWAS analysis ${ }^{30}(n=52,251)$ on the polygenic risk score generated from the Aung et al's ${ }^{13}$ XFS global dataset against patients genotyped on Illumina Mega-array chip in BioVU with about 18k ICD-9 /ICD-10 codes, accounting for age, gender, and the first 5 principal components. 


\section{DISCLOSURES:}

ERG receives an honorarium from the journal Circulation Research of the American Heart Association, as a member of the Editorial Board.

The remaining authors declare no competing interests.

\section{ACKNOWLEDGMENTS:}

We are grateful to Maria Niarchou and Tyne Fleming for comments on earlier version of the manuscript.

JH was jointly supported through grant to MAB (T32 grant 5T32EY021453), ERG and NJC.

ERG is grateful to the President and Fellows of Clare Hall, University of Cambridge for the fellowship support. ERG is also supported by a NIH Genomic Innovator Award

(R35HG010718). The content is solely the responsibility of the authors and does not necessarily represent the official views of the National Institutes of Health.

KMJ - Joseph Ellis Family and William Black Research Funds, NEI Core Grant 6P30EY08126 to Vanderbilt Vision Research Center, Unrestricted Departmental Grant from Research to Prevent Blindness, Inc., NY.

The European-Data-Set was support by the Interdisciplinary Center for Clinical Research (IZKF) at the University Hospital of the University of Erlangen-Nuremberg (project E23) to AR and USS and the Deutsche Forschungsgemeinschaft (SCHL 366/8-1) to USS.

JH, NJC and ERG jointly conceived the project. AR, US and FP managed patients ' data and tissues' samples of the three European cohorts. US, DB and FP conducted functional biological experiments. FP contributed raw genotyping data for European populations. JH performed all the statistical analysis. JH drafted the manuscript with critical input from KJ, NJC, ERG, FP \& CCK. The manuscript was approved by all authors. NJC was responsible for obtaining financial support for this study. 


\section{$\underline{\text { References }}$}

1 Schlötzer-Schrehardt U. Genetics and genomics of pseudoexfoliation syndrome/glaucoma. Middle East Afr J Ophthalmol 2011;18:30-6. https://doi.org/10.4103/0974-9233.75882.

2 Schlötzer-Schrehardt U, Naumann GOH. Ocular and Systemic Pseudoexfoliation Syndrome. Am J Ophthalmol 2006;141:921-937.e2. https://doi.org/10.1016/j.ajo.2006.01.047.

3 Rebecca M, Gayathri R, Bhuvanasundar R, Sripriya K, Shantha B, Angayarkanni N. Elastin modulation and modification by homocysteine: a key factor in the pathogenesis of Pseudoexfoliation syndrome? Br J Ophthalmol 2018:bjophthalmol-2018-312088. https://doi.org/10.1136/bjophthalmol-2018312088.

4 Vesti E, Kivelä T. Exfoliation syndrome and exfoliation glaucoma. Prog Retin Eye Res 2000;19:345-68. https://doi.org/10.1016/S1350-9462(99)00019-1.

5 Chung H, Arora S, Damji KF, Weis E. Association of pseudoexfoliation syndrome with cardiovascular and cerebrovascular disease: a systematic review and meta-analysis. Can J Ophthalmol 2018;53:36572. https://doi.org/10.1016/j.jcjo.2017.10.039.

6 Andrikopoulos GK, Alexopoulos DK, Gartaganis SP. Pseudoexfoliation syndrome and cardiovascular diseases. World J Cardiol 2014;6:847-54. https://doi.org/10.4330/wjc.v6.i8.847.

7 Pasutto F, Zenkel M, Hoja U, Berner D, Uebe S, Ferrazzi F, et al. Pseudoexfoliation syndromeassociated genetic variants affect transcription factor binding and alternative splicing of LOXL1. Nat Commun 2017;8:15466.

8 Xu F, Zhang L, Li M. Plasma homocysteine, serum folic acid, serum vitamin B12, serum vitamin B6, MTHFR and risk of pseudoexfoliation glaucoma: a meta-analysis. Graefes Arch Clin Exp Ophthalmol 2012;250:1067-74. https://doi.org/10.1007/s00417-011-1877-4.

9 Clement Cl, Goldberg I, Healey PR, Graham SL. Plasma Homocysteine, MTHFR Gene Mutation, and Open-angle Glaucoma. J Glaucoma 2009;18:.

10 AASVED H. STUDY OF RELATIVES OF PERSONS WITH FIBRILLOPATHIA EPITHELIOCAPSULARIS (PSEUDOEXFOLIATION OF THE LENS CAPSULE). Acta Ophthalmol (Copenh) 1975;53:879-86. https://doi.org/10.1111/j.1755-3768.1975.tb00406.x.

11 Gottfredsdottir MS, Sverrisson T, Musch DC, Stefansson E. Chronic Open-Angle Glaucoma and Associated Ophthalmic Findings in Monozygotic Twins and Their Spouses in Iceland. J Glaucoma 1999;8:

12 Aung T, Ozaki M, Mizoguchi T, Allingham RR, Li Z, Haripriya A, et al. A common variant mapping to CACNA1A is associated with susceptibility to exfoliation syndrome. Nat Genet 2015;47:387-92. https://doi.org/10.1038/ng.3226.

13 Aung T, Ozaki M, Lee MC, Schlötzer-Schrehardt U, Thorleifsson G, Mizoguchi T, et al. Genetic association study of exfoliation syndrome identifies a protective rare variant at LOXL1 and five new susceptibility loci. Nat Genet 2017;49:993.

14 Thorleifsson G, Magnusson KP, Sulem P, Walters GB, Gudbjartsson DF, Stefansson H, et al. Common Sequence Variants in the <em $>$ LOXL1</em> Gene Confer Susceptibility to Exfoliation Glaucoma. Science 2007;317:1397. https://doi.org/10.1126/science.1146554.

15 Krumbiegel M, Pasutto F, Schlötzer-Schrehardt U, Uebe S, Zenkel M, Mardin CY, et al. Genome-wide association study with DNA pooling identifies variants at CNTNAP2 associated with pseudoexfoliation syndrome. Eur J Hum Genet EJHG 2011;19:186-93. https://doi.org/10.1038/ejhg.2010.144.

16 Nakano M, Ikeda Y, Tokuda Y, Fuwa M, Ueno M, Imai K, et al. Novel common variants and susceptible haplotype for exfoliation glaucoma specific to Asian population. Sci Rep 2014;4:5340. 
17 Zagajewska K, Piątkowska M, Goryca K, Bałabas A, Kluska A, Paziewska A, et al. GWAS links variants in neuronal development and actin remodeling related loci with pseudoexfoliation syndrome without glaucoma. Exp Eye Res 2018;168:138-48. https://doi.org/10.1016/j.exer.2017.12.006.

18 Craig JE, Hewitt AW, McMellon AE, Henders AK, Ma L, Wallace L, et al. Rapid inexpensive genomewide association using pooled whole blood. Genome Res 2009;19:2075-80. https://doi.org/10.1101/gr.094680.109.

19 Mori K, Imai K, Matsuda A, Ikeda Y, Naruse S, Hitora-Takeshita H, et al. LOXL1 genetic polymorphisms are associated with exfoliation glaucoma in the Japanese population. Mol Vis 2008;14:1037-40.

20 Thorleifsson G, Walters GB, Hewitt AW, Masson G, Helgason A, DeWan A, et al. Common variants near CAV1 and CAV2 are associated with primary open-angle glaucoma. Nat Genet 2010;42:906-9. https://doi.org/10.1038/ng.661.

21 Chen L, Jia L, Wang N, Tang G, Zhang C, Fan S, et al. Evaluation of LOXL1 polymorphisms in exfoliation syndrome in a Chinese population. Mol Vis 2009;15:2349-57.

22 Williams SEI, Whigham BT, Liu Y, Carmichael TR, Qin X, Schmidt S, et al. Major LOXL1 risk allele is reversed in exfoliation glaucoma in a black South African population. Mol Vis 2010;16:705-12.

23 Sharma S, Martin S, Sykes MJ, Dave A, Hewitt AW, Burdon KP, et al. Biological effect of LOXL1 coding variants associated with pseudoexfoliation syndrome. Exp Eye Res 2016;146:212-23. https://doi.org/10.1016/j.exer.2016.03.013.

$24 \mathrm{Kim} \mathrm{S}$, Kim Y. Variations in LOXL1 associated with exfoliation glaucoma do not affect amine oxidase activity. Mol Vis 2012;18:265-70.

25 Berner D, Hoja U, Zenkel M, Ross JJ, Uebe S, Paoli D, et al. The protective variant rs7173049 at LOXL1 locus impacts on retinoic acid signaling pathway in pseudoexfoliation syndrome. Hum Mol Genet 2019. https://doi.org/10.1093/hmg/ddz075.

26 Hauser MA, Aboobakar IF, Liu Y, Miura S, Whigham BT, Challa P, et al. Genetic variants and cellular stressors associated with exfoliation syndrome modulate promoter activity of a IncRNA within the LOXL1 locus. Hum Mol Genet 2015;24:6552-63. https://doi.org/10.1093/hmg/ddv347.

27 Gamazon ER, Wheeler HE, Shah KP, Mozaffari SV, Aquino-Michaels K, Carroll RJ, et al. A gene-based association method for mapping traits using reference transcriptome data. Nat Genet 2015;47:10918.

28 Barbeira AN, Dickinson SP, Bonazzola R, Zheng J, Wheeler HE, Torres JM, et al. Exploring the phenotypic consequences of tissue specific gene expression variation inferred from GWAS summary statistics. Nat Commun 2018;9:1825-1825. https://doi.org/10.1038/s41467-018-03621-1.

29 Aung T, Ozaki M, Lee MC, Schlötzer-Schrehardt U, Thorleifsson G, Mizoguchi T, et al. Genetic association study of exfoliation syndrome identifies a protective rare variant at LOXL1 and five new susceptibility loci. Nat Genet 2017;49:993.

30 Wagner AH, Anand VN, Wang W-H, Chatterton JE, Sun D, Shepard AR, et al. Exon-level expression profiling of ocular tissues. Exp Eye Res 2013;111:105-11. https://doi.org/10.1016/j.exer.2013.03.004.

31 Thul PJ, Åkesson L, Wiking M, Mahdessian D, Geladaki A, Ait Blal H, et al. A subcellular map of the human proteome. Science 2017;356:eaal3321. https://doi.org/10.1126/science.aal3321.

32 Uhlén M, Fagerberg L, Hallström BM, Lindskog C, Oksvold P, Mardinoglu A, et al. Tissue-based map of the human proteome. Science 2015;347:1260419. https://doi.org/10.1126/science.1260419.

33 Chen EY, Tan CM, Kou Y, Duan Q, Wang Z, Meirelles GV, et al. Enrichr: interactive and collaborative HTML5 gene list enrichment analysis tool. BMC Bioinformatics 2013;14:128-128. https://doi.org/10.1186/1471-2105-14-128.

34 Kuleshov MV, Jones MR, Rouillard AD, Fernandez NF, Duan Q, Wang Z, et al. Enrichr: a comprehensive gene set enrichment analysis web server 2016 update. Nucleic Acids Res 2016;44:W90-7. https://doi.org/10.1093/nar/gkw377. 
35 Cornelis MC, Monda KL, Yu K, Paynter N, Azzato EM, Bennett SN, et al. Genome-wide meta-analysis identifies regions on 7p21 (AHR) and 15q24 (CYP1A2) as determinants of habitual caffeine consumption. PLoS Genet 2011;7:e1002033-e1002033.

https://doi.org/10.1371/journal.pgen.1002033.

36 Amin N, Byrne E, Johnson J, Chenevix-Trench G, Walter S, Nolte IM, et al. Genome-wide association analysis of coffee drinking suggests association with CYP1A1/CYP1A2 and NRCAM. Mol Psychiatry 2012;17:1116-29. https://doi.org/10.1038/mp.2011.101.

37 Cornelis MC, Kacprowski T, Menni C, Gustafsson S, Pivin E, Adamski J, et al. Genome-wide association study of caffeine metabolites provides new insights to caffeine metabolism and dietary caffeineconsumption behavior. Hum Mol Genet 2016;25:5472-82. https://doi.org/10.1093/hmg/ddw334.

38 Feitosa MF, Kraja AT, Chasman DI, Sung YJ, Winkler TW, Ntalla I, et al. Novel genetic associations for blood pressure identified via gene-alcohol interaction in up to $570 \mathrm{~K}$ individuals across multiple ancestries. PloS One 2018;13:e0198166-e0198166. https://doi.org/10.1371/journal.pone.0198166.

39 Sulem P, Gudbjartsson DF, Geller F, Prokopenko I, Feenstra B, Aben KKH, et al. Sequence variants at CYP1A1-CYP1A2 and AHR associate with coffee consumption. Hum Mol Genet 2011;20:2071-7. https://doi.org/10.1093/hmg/ddr086.

40 Coffee and Caffeine Genetics Consortium, Cornelis MC, Byrne EM, Esko T, Nalls MA, Ganna A, et al. Genome-wide meta-analysis identifies six novel loci associated with habitual coffee consumption. Mol Psychiatry 2015;20:647-56. https://doi.org/10.1038/mp.2014.107.

41 Li Y, Yao J, Han C, Yang J, Chaudhry MT, Wang S, et al. Quercetin, Inflammation and Immunity. Nutrients 2016;8:167-167. https://doi.org/10.3390/nu8030167.

42 Simmons DL, Wagner D, Westover K. Nonsteroidal Anti-Inflammatory Drugs, Acetaminophen, Cyclooxygenase 2, and Fever. Clin Infect Dis 2000;31:S211-8. https://doi.org/10.1086/317517.

43 Kraan J, van den Broek P, Verhoef C, Grunhagen DJ, Taal W, Gratama JW, et al. Endothelial CD276 (B7-H3) expression is increased in human malignancies and distinguishes between normal and tumour-derived circulating endothelial cells. Br J Cancer 2014;111:149.

44 Wood JJ, Boyne JR, Paulus C, Jackson BR, Nevels MM, Whitehouse A, et al. ARID3B: a Novel Regulator of the Kaposi's Sarcoma-Associated Herpesvirus Lytic Cycle. J Virol 2016;90:9543. https://doi.org/10.1128/JVI.03262-15.

45 Lin C, Song W, Bi X, Zhao J, Huang Z, Li Z, et al. Recent advances in the ARID family: focusing on roles in human cancer. OncoTargets Ther 2014;7:315-24. https://doi.org/10.2147/OTT.S57023.

46 Jeong YJ, Park SH, Mun SH, Kwak SG, Lee S-J, Oh HK. Association between lysyl oxidase and fibrotic focus in relation with inflammation in breast cancer. Oncol Lett 2018;15:2431-40. https://doi.org/10.3892/ol.2017.7617.

47 Burgoyne RD, Morgan A. Secretory Granule Exocytosis. Physiol Rev 2003;83:581-632. https://doi.org/10.1152/physrev.00031.2002.

48 Liu L, Guo Z, Tieu Q, Castle A, Castle D. Role of secretory carrier membrane protein SCAMP2 in granule exocytosis. Mol Biol Cell 2002;13:4266-78. https://doi.org/10.1091/mbc.e02-03-0136.

49 Blank U, Madera-Salcedo IK, Danelli L, Claver J, Tiwari N, Sánchez-Miranda E, et al. Vesicular trafficking and signaling for cytokine and chemokine secretion in mast cells. Front Immunol 2014;5:453-453. https://doi.org/10.3389/fimmu.2014.00453.

50 Chen X, Li Y, Blankson S, Liu M, Huang D, Redmond HP, et al. B7-H3 Augments Inflammatory Responses and Exacerbates Brain Damage via Amplifying NF-KB p65 and MAPK p38 Activation during Experimental Pneumococcal Meningitis. PLOS ONE 2017;12:e0171146. https://doi.org/10.1371/journal.pone.0171146.

51 Zhang G, Wang J, Kelly J, Gu G, Hou J, Zhou Y, et al. B7-H3 Augments the Inflammatory Response and Is Associated with Human Sepsis. J Immunol 2010;185:3677. https://doi.org/10.4049/jimmunol.0904020. 
52 Jeong YJ, Park SH, Mun SH, Kwak SG, Lee S-J, Oh HK. Association between lysyl oxidase and fibrotic focus in relation with inflammation in breast cancer. Oncol Lett 2018;15:2431-40.

https://doi.org/10.3892/ol.2017.7617.

53 Zhao W, Yang A, Chen W, Wang P, Liu T, Cong M, et al. Inhibition of lysyl oxidase-like 1 (LOXL1) expression arrests liver fibrosis progression in cirrhosis by reducing elastin crosslinking. Biochim Biophys Acta BBA - Mol Basis Dis 2018;1864:1129-37. https://doi.org/10.1016/j.bbadis.2018.01.019.

54 Bellaye P-S, Shimbori C, Upagupta C, Sato S, Shi W, Gauldie J, et al. Lysyl Oxidase-Like 1 Protein Deficiency Protects Mice from Adenoviral Transforming Growth Factor- $\beta 1$-induced Pulmonary Fibrosis. Am J Respir Cell Mol Biol 2017;58:461-70. https://doi.org/10.1165/rcmb.2017-0252OC.

55 Kumar P, Smith T, Raeman R, Chopyk DM, Brink H, Liu Y, et al. Periostin promotes liver fibrogenesis by activating lysyl oxidase in hepatic stellate cells. J Biol Chem 2018;293:12781-92. https://doi.org/10.1074/jbc.RA117.001601.

$56 \mathrm{Hu} \mathrm{H}$, Sun S-C. Ubiquitin signaling in immune responses. Cell Res 2016;26:457.

$57 \mathrm{Wu}$ Y, Kang J, Zhang L, Liang Z, Tang X, Yan Y, et al. Ubiquitination regulation of inflammatory responses through NF-KB pathway. Am J Transl Res 2018;10:881-91.

58 Corn JE, Vucic D. Ubiquitin in inflammation: the right linkage makes all the difference. Nat Struct Amp Mol Biol 2014;21:297.

59 Shetty R, Ghosh A, Lim RR, Subramani M, Mihir K, A. R R, et al. Elevated Expression of Matrix Metalloproteinase- 9 and Inflammatory Cytokines in Keratoconus Patients Is Inhibited by Cyclosporine A. Invest Ophthalmol Vis Sci 2015;56:738-50. https://doi.org/10.1167/iovs.14-14831.

60 Demarquoy J, Borgne FL. Biosynthesis, metabolism and function of protectins and resolvins. Clin Lipido/ 2014;9:683-93. https://doi.org/10.2217/clp.14.44.

61 Kohli P, Levy BD. Resolvins and protectins: mediating solutions to inflammation. Br J Pharmacol 2009;158:960-71. https://doi.org/10.1111/j.1476-5381.2009.00290.x.

62 Stark DT, Bazan NG. Neuroprotectin D1 Induces Neuronal Survival and Downregulation of Amyloidogenic Processing in Alzheimer's Disease Cellular Models. Mol Neurobiol 2011;43:131-8. https://doi.org/10.1007/s12035-011-8174-4.

63 Downie LE, Vingrys AJ. Oral Omega-3 Supplementation Lowers Intraocular Pressure in Normotensive Adults. Trans/ Vis Sci Technol 2018;7:1-1. https://doi.org/10.1167/tvst.7.3.1.

64 Huang W-B, Fan Q, Zhang X-L. Cod liver oil: a potential protective supplement for human glaucoma. Int J Ophthalmol 2011;4:648-51. https://doi.org/10.3980/j.issn.2222-3959.2011.06.15.

65 Nguyen CTO, Bui BV, Sinclair AJ, Vingrys AJ. Dietary Omega 3 Fatty Acids Decrease Intraocular Pressure with Age by Increasing Aqueous Outflow. Invest Ophthalmol Vis Sci 2007;48:756-62. https://doi.org/10.1167/iovs.06-0585.

66 Mildenberger J, Johansson I, Sergin I, Kjøbli E, Damås JK, Razani B, et al. N-3 PUFAs induce inflammatory tolerance by formation of KEAP1-containing SQSTM1/p62-bodies and activation of NFE2L2. Autophagy 2017;13:1664-78. https://doi.org/10.1080/15548627.2017.1345411.

67 Dewundara SS, Wiggs JL, Sullivan DA, Pasquale LR. Is Estrogen a Therapeutic Target for Glaucoma? Semin Ophthalmol 2016;31:140-6. https://doi.org/10.3109/08820538.2015.1114845.

68 Kitaoka Y, Munemasa Y, Hayashi Y, Kuribayashi J, Koseki N, Kojima K, et al. Axonal Protection by $17 \beta-$ Estradiol through Thioredoxin-1 in Tumor Necrosis Factor-Induced Optic Neuropathy. Endocrinology 2011;152:2775-85. https://doi.org/10.1210/en.2011-0046.

69 Caprioli J, Munemasa Y, Kwong JMK, Piri N. Overexpression of thioredoxins 1 and 2 increases retinal ganglion cell survival after pharmacologically induced oxidative stress, optic nerve transection, and in experimental glaucoma. Trans Am Ophthalmol Soc 2009;107:161-5.

70 Yildirim Z, Yildirim F, Uçgun NI, Sepici-Dinçel A. The role of the cytokines in the pathogenesis of pseudoexfoliation syndrome. Int J Ophthalmol 2013;6:50-3. https://doi.org/10.3980/j.issn.22223959.2013.01.10. 
71 Zenkel M, Krysta A, Pasutto F, Juenemann A, Kruse FE, Schlötzer-Schrehardt U. Regulation of Lysyl Oxidase-like 1 (LOXL1) and Elastin-Related Genes by Pathogenic Factors Associated with Pseudoexfoliation Syndrome. Invest Ophthalmol Vis Sci 2011;52:8488-95. https://doi.org/10.1167/iovs.11-8361.

72 Türkyılmaz K, Öner V, Kırbas A, Sevim MS, Sekeryapan B, Özgür G, et al. Serum YKL-40 levels as a novel marker of inflammation and endothelial dysfunction in patients with pseudoexfoliation syndrome. Eye Lond Engl 2013;27:854-9. https://doi.org/10.1038/eye.2013.92.

73 Gonen T, Guzel S, Keskinbora KH. YKL-40 is a local marker for inflammation in patients with pseudoexfoliation syndrome. Eye 2019;33:772-6. https://doi.org/10.1038/s41433-018-0308-8.

74 Sorkhabi R, Ghorbanihaghjo A, Ahoor M, Nahaei M, Rashtchizadeh N. High-sensitivity C-reactive Protein and Tumor Necrosis Factor Alpha in Pseudoexfoliation Syndrome. Oman Med J 2013;28:16-9. https://doi.org/10.5001/omj.2013.04.

75 Yüksel N, Pirhan D, Altntas Ö, Çaglar Y. Systemic High-sensitivity C-reactive Protein Level in Pseudoexfoliation Syndrome and Pseudoexfoliation Glaucoma. J Glaucoma 2010;19:.

76 Pasquale LR, Wiggs JL, Willett WC, Kang JH. The Relationship between caffeine and coffee consumption and exfoliation glaucoma or glaucoma suspect: a prospective study in two cohorts. Invest Ophthalmol Vis Sci 2012;53:6427-33. https://doi.org/10.1167/iovs.12-10085.

77 Grubben MJ, Boers GH, Blom HJ, Broekhuizen R, de Jong R, van Rijt L, et al. Unfiltered coffee increases plasma homocysteine concentrations in healthy volunteers: a randomized trial. Am J Clin Nutr 2000;71:480-4. https://doi.org/10.1093/ajcn/71.2.480.

78 Urgert R, van Vliet T, Zock PL, Katan MB. Heavy coffee consumption and plasma homocysteine: a randomized controlled trial in healthy volunteers. Am J Clin Nutr 2000;72:1107-10. https://doi.org/10.1093/ajcn/72.5.1107.

79 Bleich S, Roedl J, Von Ahsen N, Schlötzer-Schrehardt U, Reulbach U, Beck G, et al. Elevated homocysteine levels in aqueous humor of patients with pseudoexfoliation glaucoma. Am J Ophthalmol 2004;138:162-4. https://doi.org/10.1016/j.ajo.2004.02.027.

80 Muqaku B, Tahir A, Klepeisz P, Bileck A, Kreutz D, Mayer RL, et al. Coffee consumption modulates inflammatory processes in an individual fashion. Mol Nutr Food Res 2016;60:2529-41. https://doi.org/10.1002/mnfr.201600328.

81 Paiva C, Beserra B, Reis C, Dorea J, Da Costa T, Amato A. Consumption of coffee or caffeine and serum concentration of inflammatory markers: A systematic review. Crit Rev Food Sci Nutr 2019;59:652-63. https://doi.org/10.1080/10408398.2017.1386159.

82 Furman D, Chang J, Lartigue L, Bolen CR, Haddad F, Gaudilliere B, et al. Expression of specific inflammasome gene modules stratifies older individuals into two extreme clinical and immunological states. Nat Med 2017;23:174.

83 Rivera-Oliver M, Díaz-Ríos M. Using caffeine and other adenosine receptor antagonists and agonists as therapeutic tools against neurodegenerative diseases: a review. Life Sci 2014;101:1-9. https://doi.org/10.1016/j.Ifs.2014.01.083.

84 Berner D, Zenkel M, Pasutto F, Hoja U, Liravi P, Gusek-Schneider GC, et al. Posttranscriptional Regulation of LOXL1 Expression Via Alternative Splicing and Nonsense-Mediated mRNA Decay as an Adaptive Stress Response. Invest Ophthalmol Vis Sci 2017;58:5930-40. https://doi.org/10.1167/iovs.17-22963.

85 Bobbs A, Gellerman K, Hallas WM, Joseph S, Yang C, Kurkewich J, et al. ARID3B Directly Regulates Ovarian Cancer Promoting Genes. PloS One 2015;10:e0131961-e0131961. https://doi.org/10.1371/journal.pone.0131961.

86 Ovodenko B, Rostagno A, Neubert TA, Shetty V, Thomas S, Yang A, et al. Proteomic Analysis of Exfoliation Deposits. Invest Ophthalmol Vis Sci 2007;48:1447-57. https://doi.org/10.1167/iovs.060411. 
87 Srinivasan K, Strickland P, Valdes A, Shin GC, Hinck L. Netrin-1/Neogenin Interaction Stabilizes Multipotent Progenitor Cap Cells during Mammary Gland Morphogenesis. Dev Cell 2003;4:371-82. https://doi.org/10.1016/S1534-5807(03)00054-6.

88 Tsai $\mathrm{C}-\mathrm{H}$, Chang T-Y, Chang Y-C. Upregulation of lysyl oxidase expression in cyclosporin A-induced gingival overgrowth. J Dent Sci 2009;4:13-7. https://doi.org/10.1016/S1991-7902(09)60003-9.

89 Vahabi Surena, Salman Bahareh Nazemi, Rezazadeh Fahimeh, Namdari Mahshid. Effects of cyclosporine and phenytoin on biomarker expressions in gingival fibroblasts of children and adults: an in vitro study. J Basic Clin Physiol Pharmacol 2013;25:167. https://doi.org/10.1515/jbcpp-2013-0003.

90 Takahashi N, Hayano T, Suzuki M. Peptidyl-prolyl cis-trans isomerase is the cyclosporin A-binding protein cyclophilin. Nature 1989;337:473-5. https://doi.org/10.1038/337473a0.

91 Seet L-F, Toh LZ, Finger SN, Chu SWL, Stefanovic B, Wong TT. Valproic acid suppresses collagen by selective regulation of Smads in conjunctival fibrosis. J Mol Med Berl Ger 2016;94:321-34. https://doi.org/10.1007/s00109-015-1358-z.

92 Chu C, Deng J, Xiang L, Wu Y, Wei X, Qu Y, et al. Evaluation of epigallocatechin-3-gallate (EGCG) crosslinked collagen membranes and concerns on osteoblasts. Mater Sci Eng C 2016;67:386-94. https://doi.org/10.1016/j.msec.2016.05.021.

93 Carroll CC, Martineau K, Arthur KA, Huynh RT, Volper BD, Broderick TL. The effect of chronic treadmill exercise and acetaminophen on collagen and cross-linking in rat skeletal muscle and heart. Am J Physiol-Regul Integr Comp Physiol 2014;308:R294-9. https://doi.org/10.1152/ajpregu.00374.2014.

94 Yoon JS, Chae MK, Jang SY, Lee SY, Lee EJ. Antifibrotic Effects of Quercetin in Primary Orbital Fibroblasts and Orbital Fat Tissue Cultures of Graves' Orbitopathy. Invest Ophthalmol Vis Sci 2012;53:5921-9. https://doi.org/10.1167/iovs.12-9646.

95 Ganbold M, Shimamoto Y, Ferdousi F, Tominaga K, Isoda H. Antifibrotic effect of methylated quercetin derivatives on TGF $\beta$-induced hepatic stellate cells. Biochem Biophys Rep 2019;20:100678100678. https://doi.org/10.1016/j.bbrep.2019.100678.

96 Donejko M, Przylipiak A, Rysiak E, Głuszuk K, Surażyński A. Influence of caffeine and hyaluronic acid on collagen biosynthesis in human skin fibroblasts. Drug Des Devel Ther 2014;8:1923-8. https://doi.org/10.2147/DDDT.S69791.

97 Gressner OA. About coffee, cappuccino and connective tissue growth factor-Or how to protect your liver!? Environ Toxicol Pharmacol 2009;28:1-10. https://doi.org/10.1016/j.etap.2009.02.005.

98 Fehrholz M, Glaser K, Speer CP, Seidenspinner S, Ottensmeier B, Kunzmann S. Caffeine modulates glucocorticoid-induced expression of CTGF in lung epithelial cells and fibroblasts. Respir Res 2017;18:51. https://doi.org/10.1186/s12931-017-0535-8.

99 Tatler AL, Barnes J, Habgood A, Goodwin A, McAnulty RJ, Jenkins G. Caffeine inhibits TGF $\beta$ activation in epithelial cells, interrupts fibroblast responses to TGF $\beta$, and reduces established fibrosis in <em>ex vivo</em> precision-cut lung slices. Thorax 2016;71:565. https://doi.org/10.1136/thoraxjnl-2015208215.

100 Cataldo A, Hamilton D, Barnett J, Paskevich P, Nixon R. Properties of the endosomal-lysosomal system in the human central nervous system: disturbances mark most neurons in populations at risk to degenerate in Alzheimer's disease. J Neurosci 1996;16:186.

https://doi.org/10.1523/JNEUROSCl.16-01-00186.1996.

101 Taraboulos A, Raeber AJ, Borchelt DR, Serban D, Prusiner SB. Synthesis and trafficking of prion proteins in cultured cells. Mol Biol Cell 1992;3:851-63. https://doi.org/10.1091/mbc.3.8.851.

102 Kegel KB, Kim M, Sapp E, McIntyre C, Castaño JG, Aronin N, et al. Huntingtin Expression Stimulates Endosomal-Lysosomal Activity, Endosome Tubulation, and Autophagy. $J$ Neurosci 2000;20:7268. https://doi.org/10.1523/JNEUROSCI.20-19-07268.2000. 
103 Kumar P, Smith T, Raeman R, Chopyk DM, Brink H, Liu Y, et al. Periostin promotes liver fibrogenesis by activating lysyl oxidase in hepatic stellate cells. J Biol Chem 2018;293:12781-92. https://doi.org/10.1074/jbc.RA117.001601.

104 Laczko R, Szauter KM, Csiszar K. LOXL1-associated candidate epithelial pathomechanisms in exfoliation glaucoma. J Glaucoma 2014;23:S43-7. https://doi.org/10.1097/IJG.0000000000000105.

105 Zenkel M, Lewczuk P, Jünemann A, Kruse FE, Naumann GOH, Schlötzer-Schrehardt U. Proinflammatory cytokines are involved in the initiation of the abnormal matrix process in pseudoexfoliation syndrome/glaucoma. Am J Pathol 2010;176:2868-79. https://doi.org/10.2353/ajpath.2010.090914.

106 Wu B, Li Y, An C, Jiang D, Gong L, Liu Y, et al. High resolution profile of body wide pathological changes induced by abnormal elastin metabolism in Loxl1 knockout mice. BioRxiv 2018:353169. https://doi.org/10.1101/353169.

107 Donley D, Nelson R, Gigley J, Fox J. Mutant huntingtin protein alters the response of microglial cells to inflammatory stimuli. BioRxiv 2019:550913. https://doi.org/10.1101/550913.

108 Currais A, Quehenberger O, M Armando A, Daugherty D, Maher P, Schubert D. Amyloid proteotoxicity initiates an inflammatory response blocked by cannabinoids. Npj Aging Mech Dis 2016;2:16012.

109 Nagaoka H, Mochida Y, Atsawasuwan P, Kaku M, Kondoh T, Yamauchi M. 1,25(OH)2D3 regulates collagen quality in an osteoblastic cell culture system. Biochem Biophys Res Commun 2008;377:6748. https://doi.org/10.1016/j.bbrc.2008.10.036.

110 Ongen H, Brown AA, Delaneau O, Panousis NI, Nica AC, GTEx Consortium, et al. Estimating the causal tissues for complex traits and diseases. Nat Genet 2017.

111 Erkelens MN, Mebius RE. Retinoic Acid and Immune Homeostasis: A Balancing Act. Trends Immunol 2017;38:168-80. https://doi.org/10.1016/j.it.2016.12.006.

112 Kim CH. Chapter four - Retinoic Acid, Immunity, and Inflammation. In: Litwack G, editor. Vitam. Horm., vol. 86. Academic Press; 2011. p. 83-101.

113 Pino-Lagos K, Guo Y, Noelle RJ. Retinoic acid: a key player in immunity. BioFactors Oxf Engl 2010;36:430-6. https://doi.org/10.1002/biof.117.

114 Gamazon ER, Segrè AV, van de Bunt M, Wen X, Xi HS, Hormozdiari F, et al. Using an atlas of gene regulation across 44 human tissues to inform complex disease- and trait-associated variation. Nat Genet 2018;50:956-67. https://doi.org/10.1038/s41588-018-0154-4.

115 Barbeira AN, Pividori M, Zheng J, Wheeler HE, Nicolae DL, Im HK. Integrating predicted transcriptome from multiple tissues improves association detection. PLOS Genet 2019;15:e1007889. https://doi.org/10.1371/journal.pgen.1007889.

116 Aguet F, Brown AA, Castel SE, Davis JR, Mohammadi P, Segrè AV, et al. Local genetic effects on gene expression across 44 human tissues. BioRxiv 2016:074450. https://doi.org/10.1101/074450.

117 Wainberg M, Sinnott-Armstrong N, Mancuso N, Barbeira AN, Knowles D, Golan D, et al. Transcriptome-wide association studies: opportunities and challenges. BioRxiv 2018:206961. https://doi.org/10.1101/206961.

118 Machiela MJ, Chanock SJ. LDlink: a web-based application for exploring population-specific haplotype structure and linking correlated alleles of possible functional variants. Bioinformatics 2015;31:3555-7. https://doi.org/10.1093/bioinformatics/btv402.

119 Machiela MJ, Chanock SJ. LDassoc: an online tool for interactively exploring genome-wide association study results and prioritizing variants for functional investigation. Bioinformatics 2017;34:887-9. https://doi.org/10.1093/bioinformatics/btx561.

120 Lin D-Y, Tao R, Kalsbeek WD, Zeng D, Gonzalez F II, Fernández-Rhodes L, et al. Genetic Association Analysis under Complex Survey Sampling: The Hispanic Community Health Study/Study of Latinos. Am J Hum Genet n.d.;95:675-88. https://doi.org/10.1016/j.ajhg.2014.11.005. 
121 Liao Y, Wang J, Jaehnig EJ, Shi Z, Zhang B. WebGestalt 2019: gene set analysis toolkit with revamped UIs and APIs. Nucleic Acids Res 2019;47:W199-205. https://doi.org/10.1093/nar/gkz401.

122 Wang J, Vasaikar S, Shi Z, Greer M, Zhang B. WebGestalt 2017: a more comprehensive, powerful, flexible and interactive gene set enrichment analysis toolkit. Nucleic Acids Res 2017;45:W130-7. https://doi.org/10.1093/nar/gkx356.

123 Wang J, Duncan D, Shi Z, Zhang B. WEB-based GEne SeT AnaLysis Toolkit (WebGestalt): update 2013. Nucleic Acids Res 2013;41:W77-83. https://doi.org/10.1093/nar/gkt439.

124 Zhang B, Kirov S, Snoddy J. WebGestalt: an integrated system for exploring gene sets in various biological contexts. Nucleic Acids Res 2005;33:W741-8. https://doi.org/10.1093/nar/gki475.

125 Croft D, Mundo AF, Haw R, Milacic M, Weiser J, Wu G, et al. The Reactome pathway knowledgebase. Nucleic Acids Res 2014;42:D472-7. https://doi.org/10.1093/nar/gkt1102.

126 Aguiar VRC, César J, Delaneau O, Dermitzakis ET, Meyer D. Expression estimation and eQTL mapping for HLA genes with a personalized pipeline. PLoS Genet 2019;15:e1008091-e1008091. https://doi.org/10.1371/journal.pgen.1008091.

127 Said I, Byrne A, Serrano V, Cardeno C, Vollmers C, Corbett-Detig R. Linked genetic variation and not genome structure causes widespread differential expression associated with chromosomal inversions. Proc Natl Acad Sci 2018;115:5492. https://doi.org/10.1073/pnas.1721275115.

128 Berner D, Zenkel M, Pasutto F, Hoja U, Liravi P, Gusek-Schneider GC, et al. Posttranscriptional Regulation of LOXL1 Expression Via Alternative Splicing and Nonsense-Mediated mRNA Decay as an Adaptive Stress ResponseLOXL1 Regulation by NMD-Coupled Alternative Splicing. Invest Ophthalmol Vis Sci 2017;58:5930-40. https://doi.org/10.1167/iovs.17-22963.

129 Denny JC, Ritchie MD, Basford MA, Pulley JM, Bastarache L, Brown-Gentry K, et al. PheWAS: demonstrating the feasibility of a phenome-wide scan to discover gene-disease associations. Bioinformatics 2010;26:1205-10. https://doi.org/10.1093/bioinformatics/btq126. 


\section{Figure legends}

Figure 1: Manhattan plot for GWAS meta-analysis and PrediXcan analysis of the genotyping data for XFS. a) The lower half of the plot is for the XFS meta-analysis summary statistics data Aung et al., 2017, while the upper half of the plot shows results from PrediXcan analysis for 48 GTEx tissues. On the $\mathrm{X}$ axis is plot of variant/gene associations along the chromosomes, while $\mathrm{Y}$ axis represent the significance levels for the associations. The legend for PrediXcan analysis on the 48 GTEx tissues, a color for each tissue, is on the right. For both plots the blue dotted line is the "suggestive" genome-wide significant threshold $(\mathrm{p}<1 \mathrm{e}-4)$, while the red line is the genome-wide significant threshold. On the lower plot, the gene labels are for genes reported/mapped to genome-wide significant signals in GWAS result, while in the upper plot is for genes that are associated at "suggestive" significant threshold. b) genes in the region in chromosome 15 that show significant association. P values for the plot in Suppl. Table S2. C15orf59 is INSYN1.

Figure 2: Conditional analysis to prioritize XFS associated genes: a) Manhattan plot for PrediXcan analysis of European ancestry individuals in tissues with predicted gene expression for a) i) LOXL1 and ii) conditioned on LOXL1 predicted gene expressions b) Manhattan plot for PrediXcan analysis of European ancestry individuals in tissues with predicted gene expression for i) STOML1 and ii) conditioned on STOML1 predicted gene expressions c) correlation in gene expression in for genes in chr15q22-25 in lung tissue for i) reference GTEx data ii) predicted gene expression in BioVU cohort. In each case on the $\mathrm{X}$ axis is plot of variant/gene associations along the chromosomes, while $\mathrm{Y}$ axis represent the significance levels for the associations. The legend for PrediXcan analysis on the GTEx tissues, a color for each tissue, is on the right. For both plots the blue dotted line is the "suggestive" genome-wide significant threshold $(\mathrm{p}<1 \mathrm{e}-4)$, while the red line is the genome-wide significant threshold. On the lower plot, the gene labels are for genes reported/mapped to genome-wide significant signals in GWAS result, while in the upper plot is for genes that are associated at "suggestive" significant threshold. $\mathrm{P}$ values for the plot in Suppl. Table S2. d) linkage disequilibrium between variants in prediction models for LOXL1 and neighboring genes in lung tissue based on pairwise $r^{2}$. C15orf59 is INSYN1.

Figure 3: Expression of NEO1, CD276, INSYN1, LOXL1, STOML1, UBL7, ARID3B and SCAMP2 mRNA in iris tissues derived from normal human donors (control) $(n=19)$ and donors with XFS syndrome $(\mathrm{n}=12)$ using real-time PCR technology. Expression levels were reduced in XFS specimens compared to control specimens, with significant differences for NEO1, CD276, LOXL1, UBL7, ARID3B and SCAMP2. The relative expression levels were normalized relative to GAPDH and are represented as mean values \pm $\mathrm{SD}(* p<0.05 ; * * p<0.01, * * * p<0.001)$.

\section{Table 1: Genes associated with XFS and replicated in European Ancestry individuals}

Table 2: Prioritization of genes that are associated with XFS: Each gene pair was checked if they had correlated predicted and expressed gene expressions, and LD checked for variants included in prediction models

Table 3: Enrichment analysis of genes that are associated with XFS 


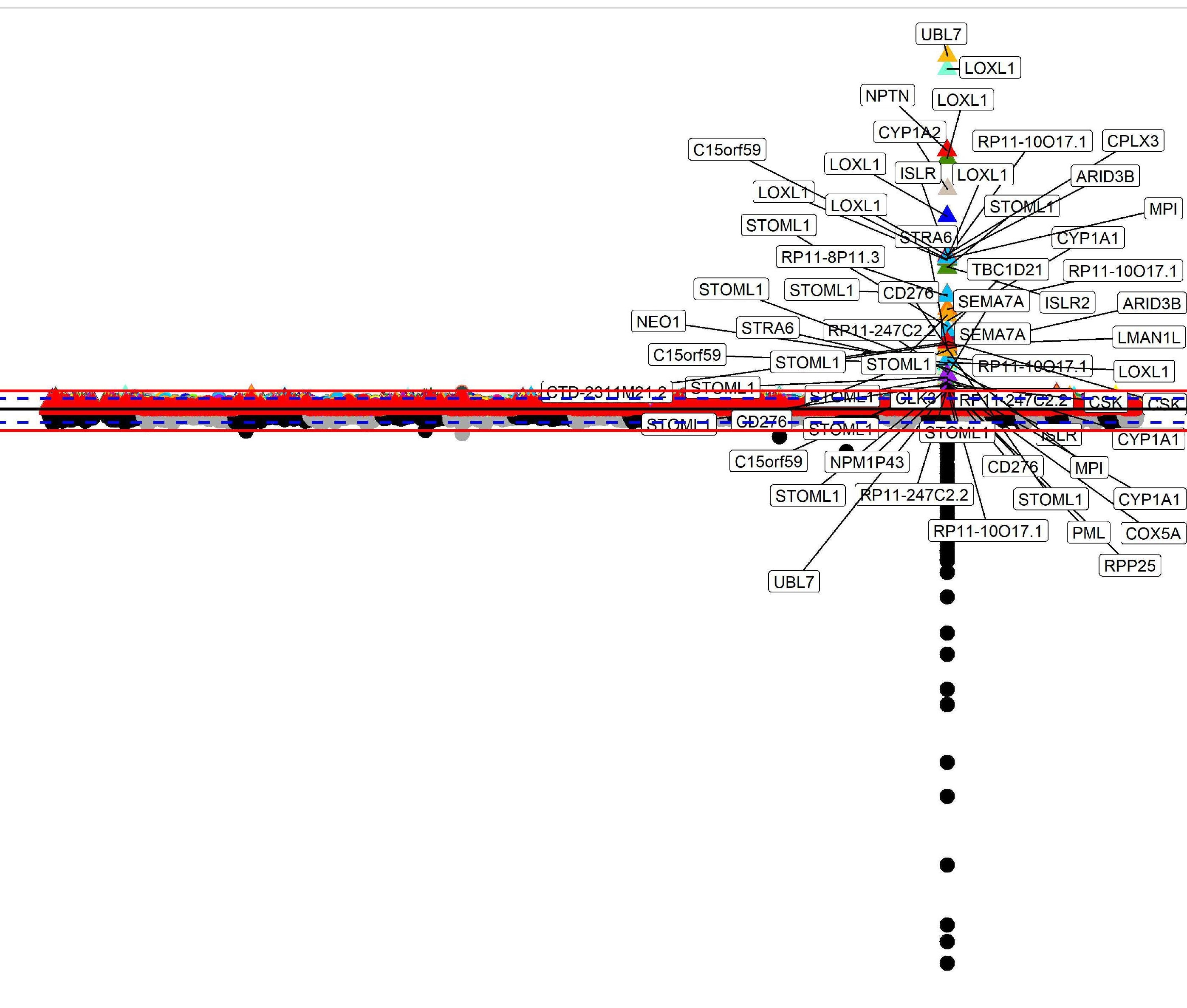

Figure 1: Manhattan plot for GWAS meta-analysis and PrediXcan analysis of the genotyping data for XFS

Tissue Type

Whole_Blood

Vagin

Uterus
Thyroid

Testis

Stomach

Spleen

Small_Intestine_Terminal_lleum

Skin_Sun_Exposed_(Lower_leg)

Skin_Not_Sun_Exposed_(Suprapubic)

Prostate

Pituitary

Pancreas

Ovary

Nerve_Tibial

Muscle_Skeletal

Minor_salivary_gland
Lung

Heart_Left_Ventricle

Hear__Atrial_Appendag

Esophagus_Muscularis

Esophagus_Mucosa

Esophagus_Gastroesophageal_Junctio

Colon_Transverse

Colon_sigmoid

Cells_Transformed_fibroblasts

Cells_EBV-transformed_lymphocytes

Breast_Mammary_Tissue

Brain_Substantia_nigra

Brain_spinal_cord

Brain_Putamen_(basal_ganglia)

Brain_Nucleus_accumb
Brain_Hippocampus

Brain_Frontal_Cortex_(BA9)

Brain_Cortex

Brain_Cerebellum

Brain_Cerebellar_Hemisphere

Brain_Caudate_(basal_ganglia)

Brain_Anterior_cingulate_cortex_(BA24) Brain_amygdala

Artery_Tibial

Artery_Coronary

Artery_Aorta

Adrenal_Gland

Adipose_Visceral_(Omentum)

Adipose_Subcutaneous

Single SNP 
Figure 1b whole BloodUterus -

Thyroid -

Testis -

Stomach -

Spleen -

Small_Intestine_Terminal_lleum -

Skin_Sun_Exposed_Lower_leg-

Skin_Not_Sun_Exposed_Suprapubic-

Prostate -

Pituitary -

Pancreas -

Ovary -

Nerve_Tibial-

Muscle_Skeletal -

Minor_Salivary_Gland -

Lung -

Liver -

Heart_Left_Ventricle -

Heart_Atrial_Appendage -

Esophagus_Muscularis -

Esophagus_Mucosa-

Esophagus_Gastroesophageal_Junction -

Colon_Transverse -

Colon_Sigmoid -

Cells_Transformed_fibroblasts -

Cells_EBV-transformed_lymphocytes -

Breast_Mammary_Tissue -

Brain_Substantia_nigra-

Brain_Putamen_basal_ganglia Brain_Nucleus_accumbens_basal_ganglia -

Brain_Hypothalamus -

Brain_Hippocampus -

Brain_Frontal_Cortex_BA9

Brain_Cortex -

Brain Cerebellum -

Brain_Cerebellar_Hemisphere -

Brain_Caudate_basal_ganglia -

Brain_Anterior_cingulate_cortex_BA24 -

Brain_Amygdala -

Artery_Tibial -

Artery_Coronary -

Artery_Aorta -

Adrenal_Gland -

Adipose_Visceral_Omentum -

Adipose_Subcutaneous -

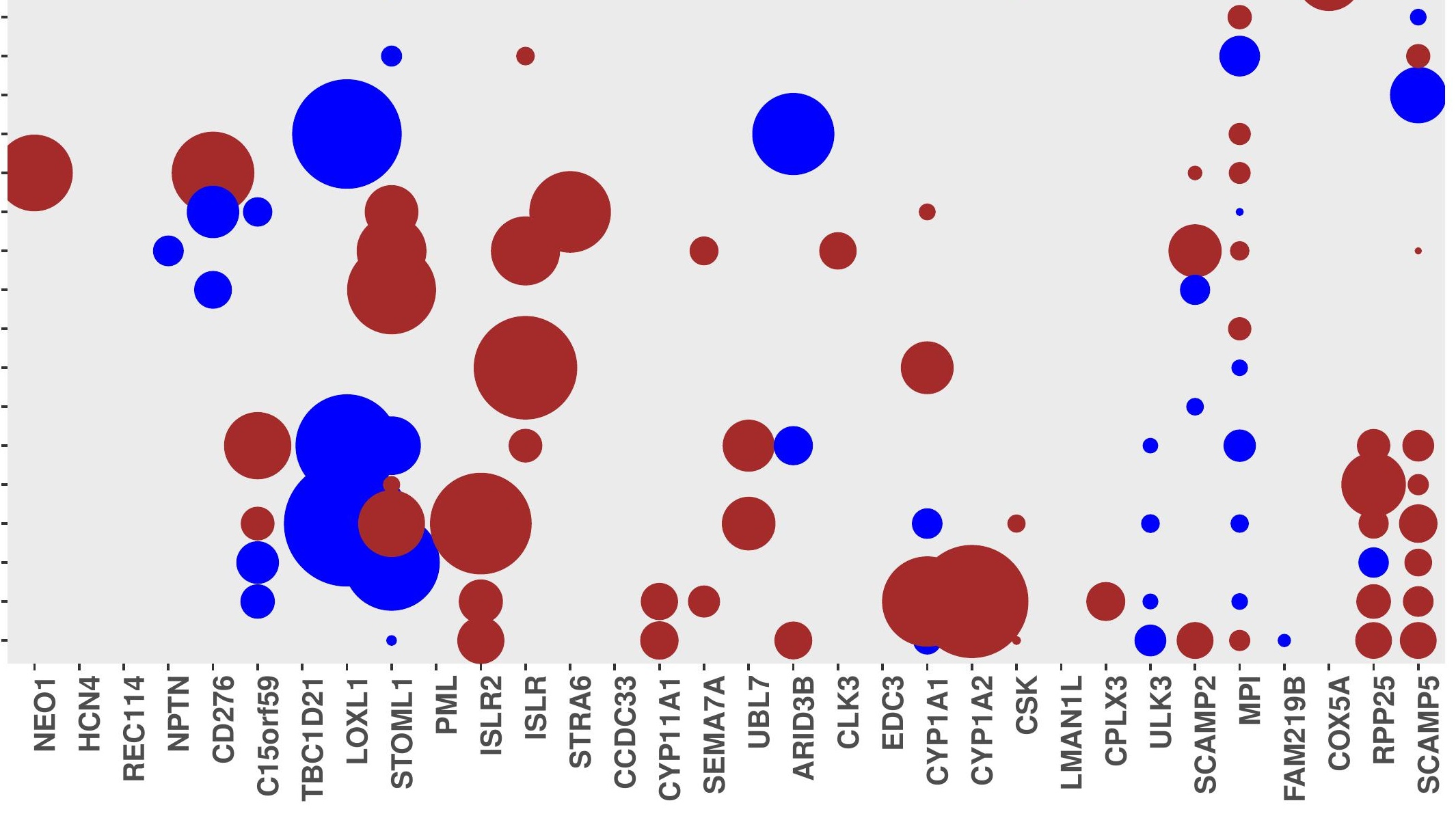


Figure 2ai: Manhattan plot of PrediXcan analysis of European ancestry individuals in tissues with predicted gene expression for LOXL1

LOXL1
LOH

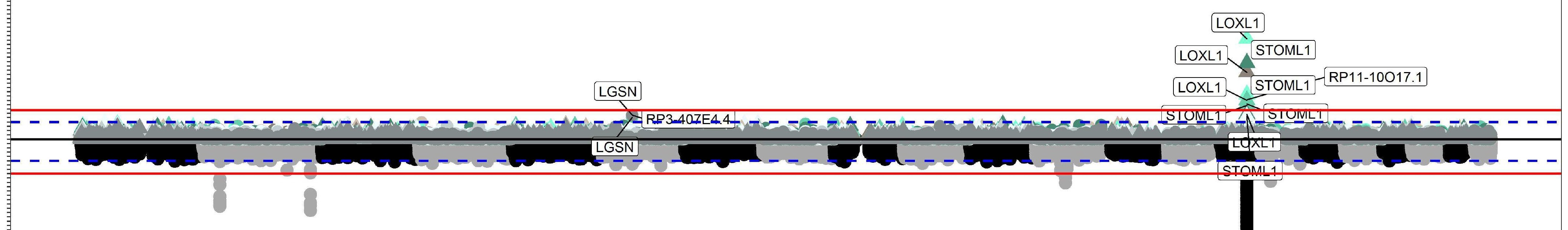

LOXL1 STOML1

Tissue Type

Prostate

Pituitary

Nerve_Tibial

Muscle

Lung

Cells_Transformed_fibroblasts

Brain_Hippocampus

Artery_tibial

Artery_Aorta

Single SNP

Single SNP

웅

0

$$
0
$$


LGSN

RP11-813P10.2

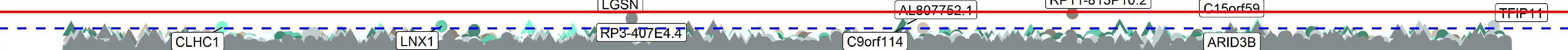
:-

lung

Hippocampus
Fibroblasts

Artery_tibial

Artery_Aorta

- Single SNP

single SNP

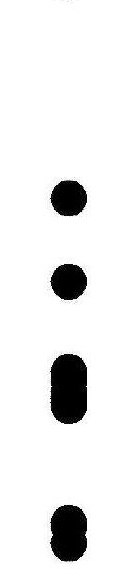


STOML1 STOML1 C15orf59 STOMLL MPI RP11-10017.1

C15orf59 STDELT LMAN1L LOXL1 STOML1 1 SEMA7A STOML1 IGSN

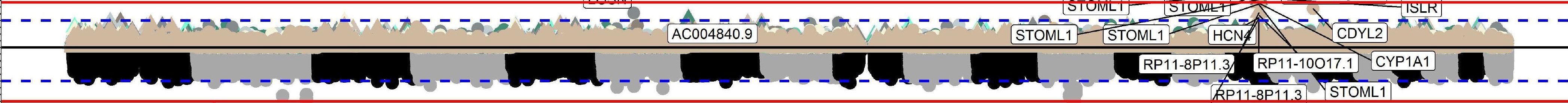

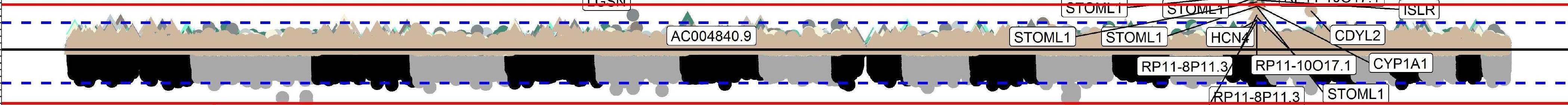

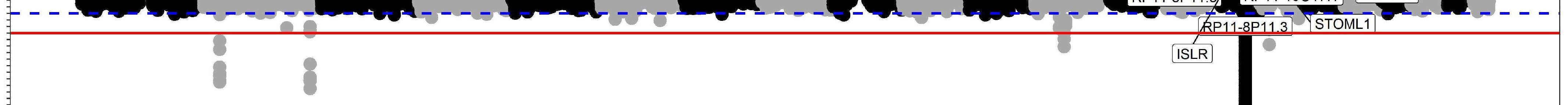

Tissue Type

Skin_Sun_Exposed_(Lower_leg) Pancreas

Lung

Heart_Left_Ventricle

Hear__Atrial_Appendage

Esophagus_Mucosa

Colon_Transverse

Cells_Transformed_fibroblasts

Brain_Cerebellum

Artery_Aorta

Adipose_Subcutaneous

Single SNP

Single SNP 
Figure 2bii: Manhattan plot for PrediXcan analysis of European ancestry individuals conditioned on STOML1 predicted gene expressions

:
LOXL1

CYP1A2
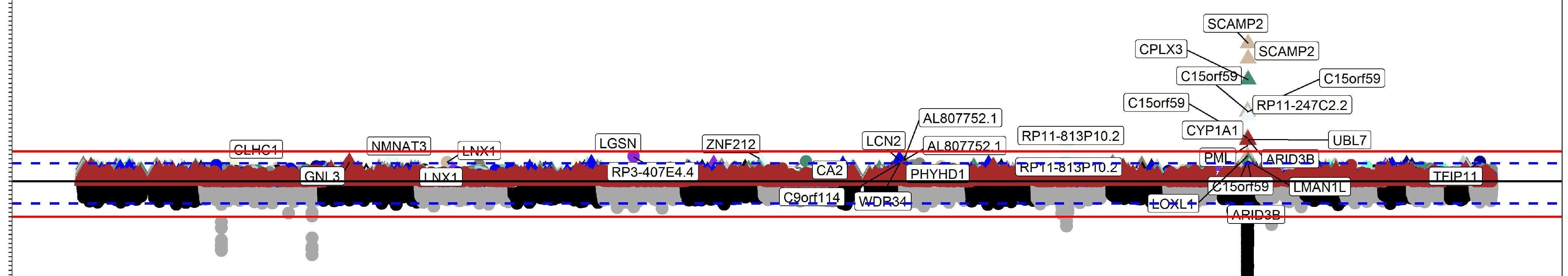

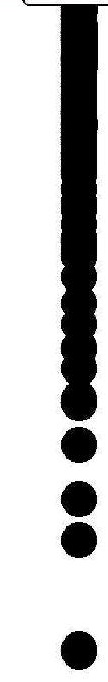

Tissue Type

skinlowerleg

prostate

pitutitary

pancreas

muscle

lung

Hippocampus

heartleftventricle

heartatrial

Fibroblasts

Colon_transverse

Cerebellum

Artery_tibial

Artery_Aorta

subcutenous

Single SNP 
Figure 2ci: correlation in measured gene expression for chr15q22-25 genes in the lung

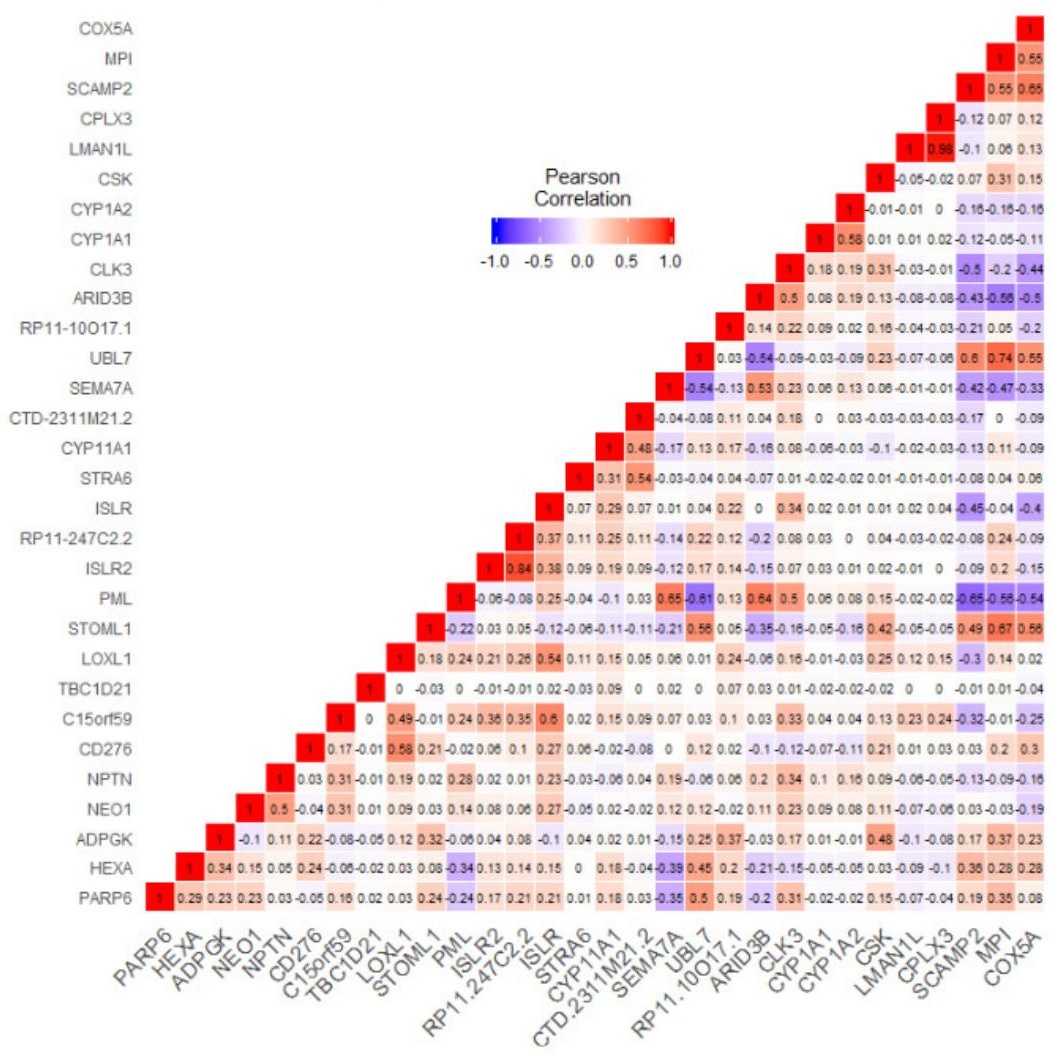


Figure 2cii: correlation in predicted gene expression for chr15q22-25 genes in lung tissue

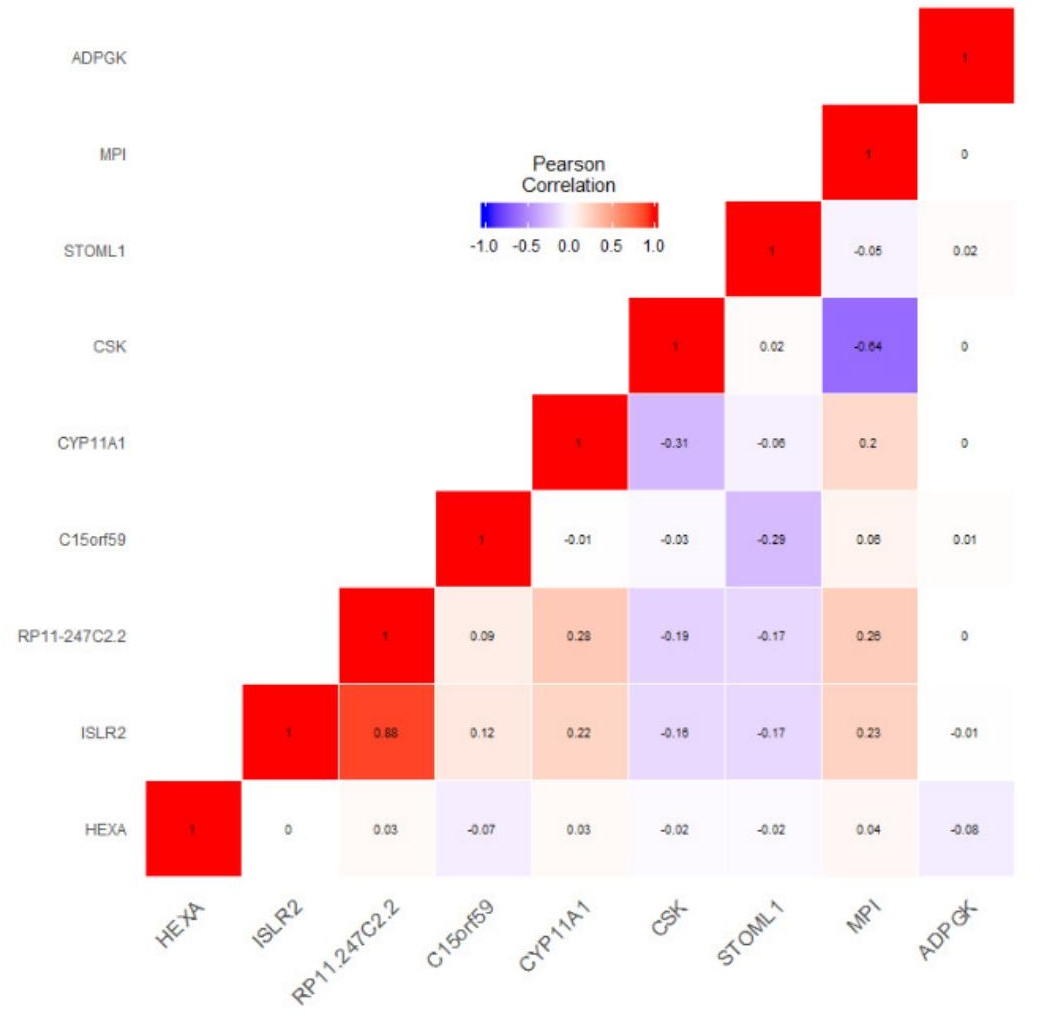


Figure 2d: Linkage disequilibrium between variants in LOXL1 and neighboring genes based on pairwise r2

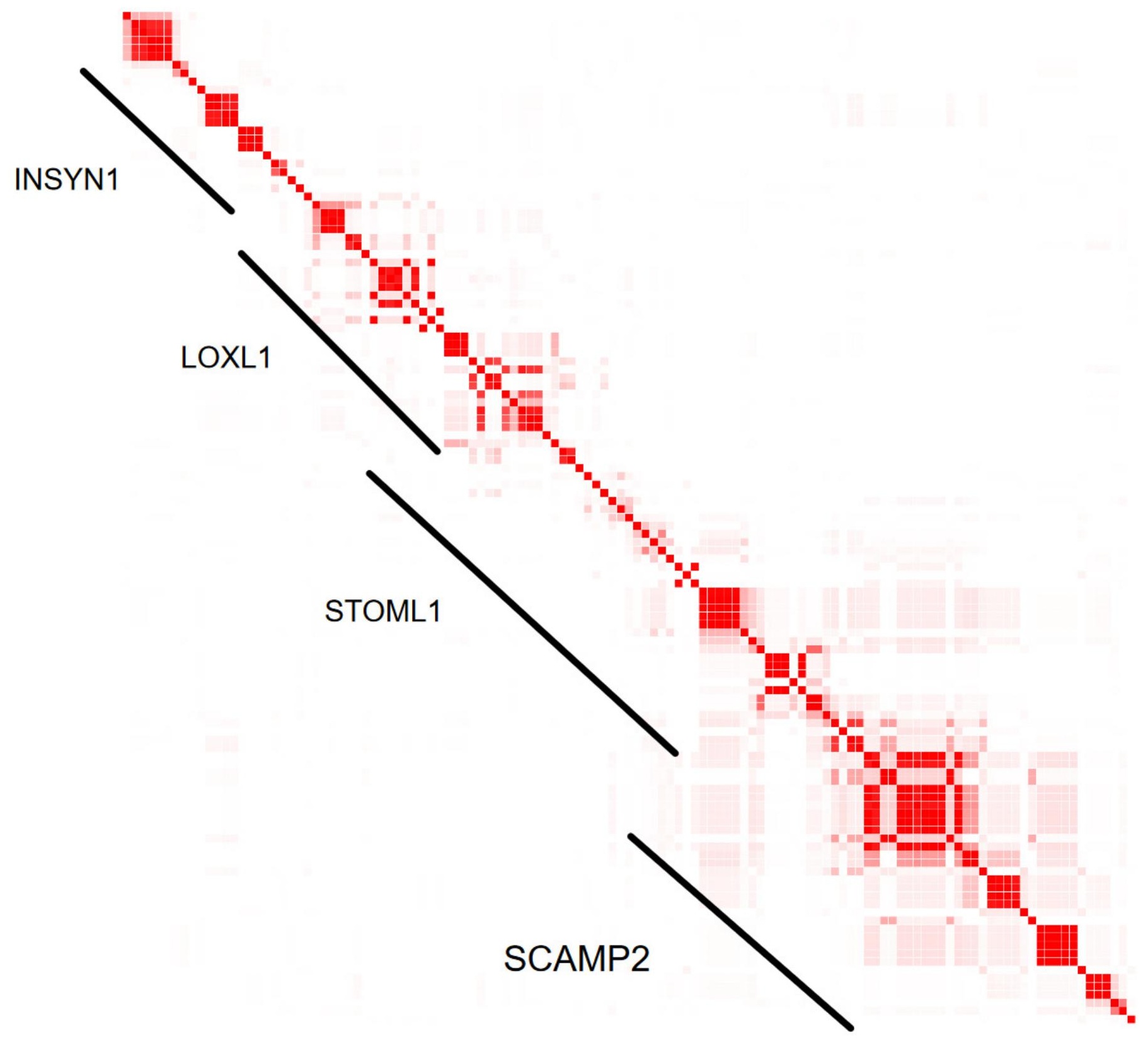


Figure 3: Gene Expression differences in iris tissues derived from normal human donors (control) and donors with XFS syndrome

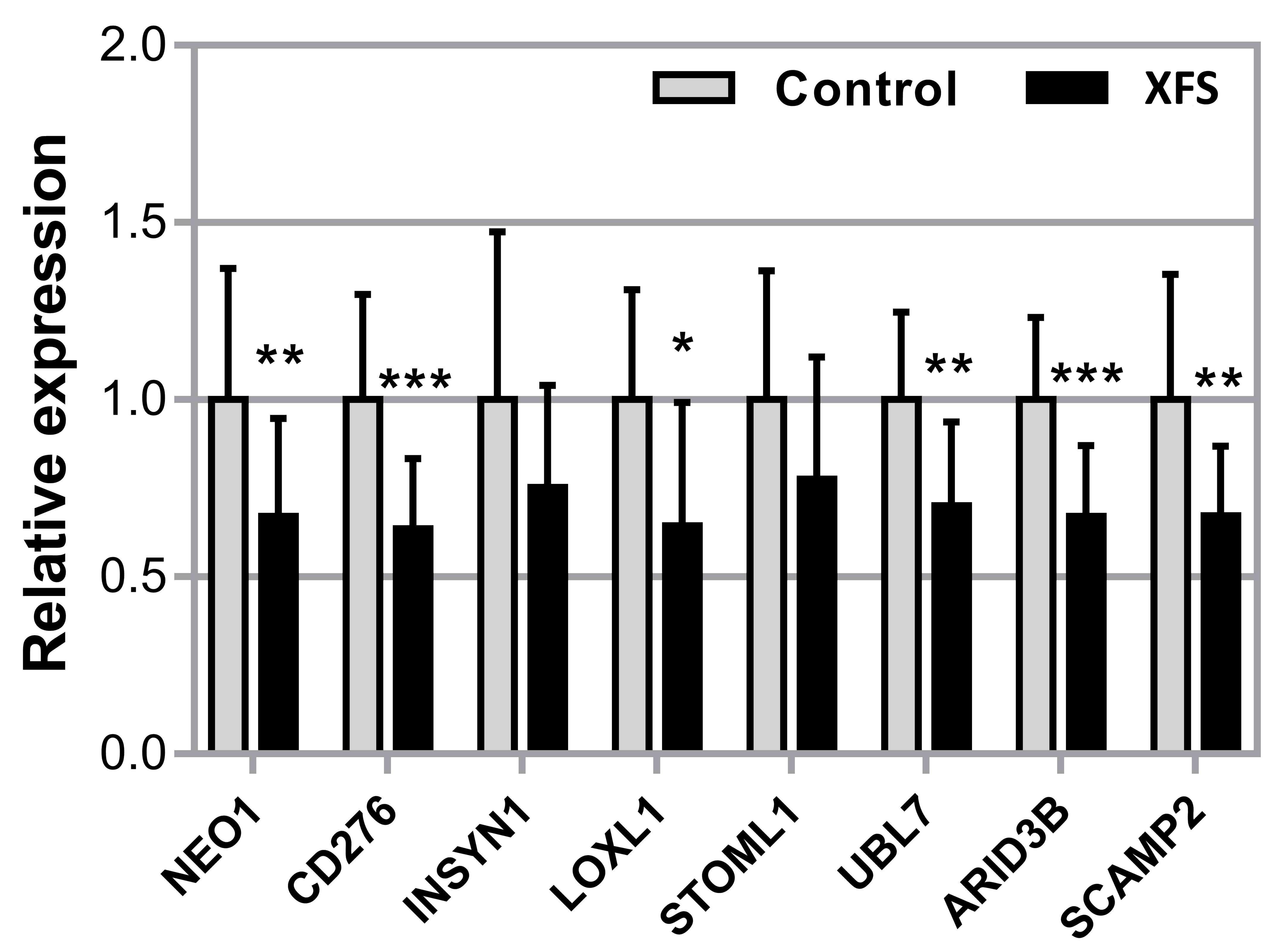


Table 1: Replication of PrediXcan analysis of global dataset in European ancestry individuals

\section{Chromosome Genes associated with XFS}

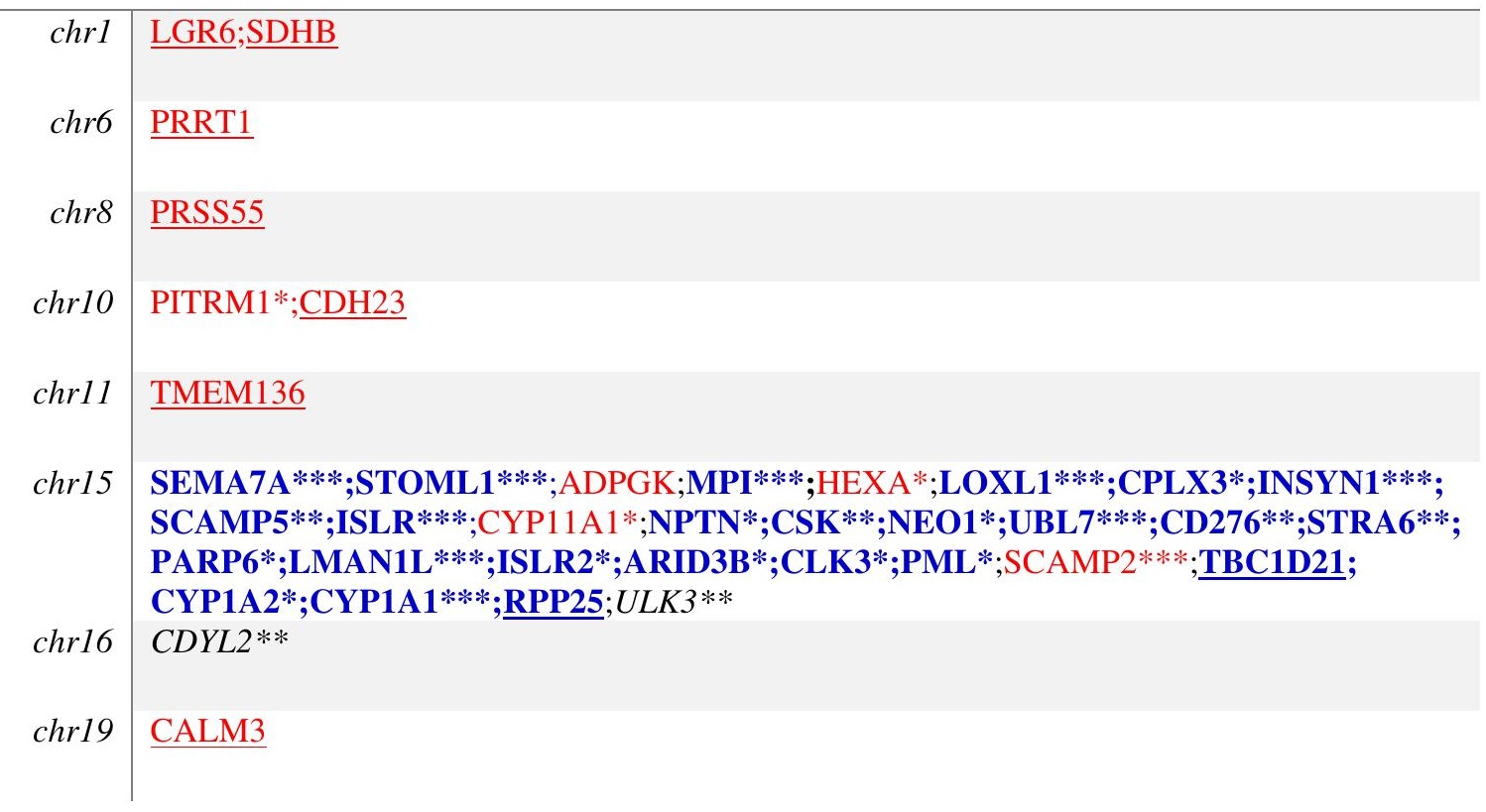

Gene associated with XFS in single tissues analysis at genome-wide significance threshold $(<2.02 e-7)$ in global GWAS summary statistic

Gene associated with XFS in cross-tissue analysis $(<9.5 e-6)$ and in single tissues analysis at suggestive significance threshold $(<1 \mathrm{e}-4)$ in global GWAS summary statistics.

Significance values in European ancestry data single tissue analysis of genes associated with XFS $* * *$ pvalue $<2 . .02 \mathrm{e} 7, * *$ pvalue $<1 \mathrm{e}-4, *$ pvalue $<0.05$

Underlined genes are those associated with XFS in global dataset but no association in European ancestry data at even nominal threshold $(<0.05)$

Additional genes associated with XFS in cross-tissue analysis of European ancestry data but not in global dataset. 
Table 2: Prioritization of genes that are associated with XFS

\begin{tabular}{|c|c|c|c|c|c|c|c|}
\hline \multirow{2}{*}{$\begin{array}{l}\text { Tissues } \\
\text { Lung }\end{array}$} & \multirow{2}{*}{$\begin{array}{l}\text { Significant Genes }^{1} \\
\text { Genes (pvalue) }\end{array}$} & \multicolumn{2}{|l|}{ Conditional Analysis } & \multirow{2}{*}{$\begin{array}{l}\text { Correlated } \\
\text { expression }\left(r^{2} \geq\right. \\
\pm 0.2)\end{array}$} & \multirow[t]{2}{*}{$\begin{array}{l}\text { LD between SNPs } \\
\text { in Prediction } \\
\text { models }(\mathbf{r} 2>0.3)+\end{array}$} & \multirow{2}{*}{$\begin{array}{l}\text { LD between } \\
\text { SNPs in } \\
\text { Prediction } \\
\text { models }(\mathrm{r} 2>0.3) \mathrm{y} \\
\end{array}$} & \multirow[t]{2}{*}{$\begin{array}{l}\text { Prediction } \\
\text { models share } \\
\text { variants }\end{array}$} \\
\hline & & LOXL1 & STOML1 & & & & \\
\hline & LOXL1 (8.75e-10) & SCAMP2 (2.73e-28) & SCAMP2 (1.01e-31) & Yes & No & LOXL1 (q) & 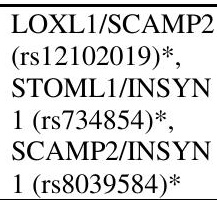 \\
\hline & STOML1 (6.60e-9) & INSYN1 (1.91e-10) & & Yes* & No & & \\
\hline \multirow[t]{3}{*}{ Brain Cerebellum } & Genes (pvalue) & STOML1 & $I S L R$ & No & Yes (s2) & & $\begin{array}{l}\text { rs12903734, } \\
\text { rs17773784 }\end{array}$ \\
\hline & STOML1 (1.48E-11) & NS & NS & & & & \\
\hline & ISLR (3.65E-08) & & & & & $\operatorname{ISLR}(\mathrm{p}, \mathrm{q})$ & \\
\hline \multirow[t]{3}{*}{ Heart atrial appendage } & Genes (pvalue) & STOML1 & INSYNI & Yes & No & & No \\
\hline & INSYN1 (4.20E-16) & NS & STOML1 (2.57E-12) & & & & \\
\hline & STOML1 (3.72E-10) & & & & & & \\
\hline \multirow[t]{3}{*}{ Colon transverse } & Genes (pvalue) & STOML1 & & No & Yes (s2) & & No \\
\hline & LMANL1 (5.61e-10) & NS & & & & & \\
\hline & STOML1 (5.54e-9) & & & & & STOML1 (p, q) & \\
\hline \multirow[t]{3}{*}{ Heart left ventricle } & Genes (pvalue) & $M P I$ & STOML1 & Yes & Yes (s2) & & $\begin{array}{l}\text { STOML1/MPI } \\
\text { (rs12442054)* }\end{array}$ \\
\hline & MPI (7.11E-17) & UBL7 (1.56E-16) & UBL7 (3.89E-10) & Yes* & Yes (s2) & MPI (p) & \\
\hline & STOML1 (3.98E-13) & & & & & & \\
\hline \multirow[t]{3}{*}{ Esophagus_Muscularis } & Genes (pvalue) & CYP1A1 & $U B L 7$ & No & No & & No \\
\hline & CYP1A1 (9.12E-11) & STOML1 (2.88E-97) & STOML1 (2.02E-98) & No & $\begin{array}{l}\text { Yes } \\
\text { (STOML1/NEO1) }\end{array}$ & STOML1 (q) & No \\
\hline & UBL7 (2.27E-08) & NEO1 (3.57E-09) & NEO1 (5.34E-09) & No* & No & & No \\
\hline
\end{tabular}

${ }^{1}$ Genes associated with XFS in European dataset at genome-wide threshold $(>2.02 \mathrm{e}-7)$

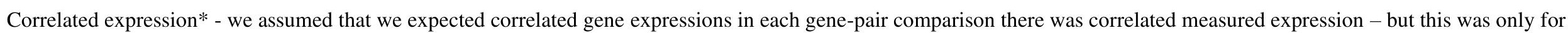
three cases out of the 3 out of eleven checked

+LD between variants in model of gene pairs

yLD between variants in a model of gene and one of the coding variants (p) rs1048661- rs4886776 (q) rs3825942

NS Not significantly associated at genome-wide threshold 
Table 3: Enrichment analysis of genes associated with XFS

\begin{tabular}{|c|c|c|c|c|c|c|}
\hline Tool & Database & enrichment & Name & \# found & \# total & Adj-p-values/FDR \\
\hline Reactome & reactome & pathway & Endosomal/Vacuolar pathway & 59 & 82 & $1.83 \mathrm{E}-07$ \\
\hline GSEA & reactome & pathway & Crosslinking of collagen fibrils & 5 & 8 & 0.031 \\
\hline \multirow[t]{4}{*}{ Enrichr } & $\begin{array}{l}\text { Jensen } \\
\text { Diseases }\end{array}$ & Disease & Rheumatoid_arthritis & 119 & 310 & $8.32 \mathrm{E}-08$ \\
\hline & & & Type_1_diabetes_mellitus & 68 & 158 & $2.69 \mathrm{E}-06$ \\
\hline & & & Carcinoma & 2619 & 11318 & 0.013 \\
\hline & & & Vitiligo & 28 & 63 & 0.029 \\
\hline Enrichr & $\begin{array}{l}\text { GWAS } \\
\text { Catalog }\end{array}$ & traits & Caffeine consumption & 11 & 14 & 0.019 \\
\hline \multirow[t]{10}{*}{ Enrichr } & DSigDB & Drugs & cyclosporin A_CTD_00007121 & 1258 & 4826 & $9.66 \mathrm{E}-11$ \\
\hline & & & VALPROIC ACID_CTD_00006977 & 2041 & 8313 & $1.75 \mathrm{E}-09$ \\
\hline & & & Copper sulfate_CTD_00007279 & 1508 & 6017 & 3.04E-08 \\
\hline & & & quercetin_CTD_00006679 & 812 & 3159 & $7.82 \mathrm{E}-05$ \\
\hline & & & acetaminophen_CTD_00005295 & 1017 & 4136 & 0.007 \\
\hline & & & AFLATOXIN B1_CTD_00007128 & 773 & 3082 & 0.006 \\
\hline & & & (-)-Epigallocatechin gallate_CTD_00002033 & 546 & 2115 & 0.005 \\
\hline & & & POTASSIUM CHROMATE_CTD_00001284 & 491 & 1898 & 0.011 \\
\hline & & & METHAMPHETAMINE_CTD_00006286 & 40 & 102 & 0.030 \\
\hline & & & $\begin{array}{l}\text { METHYL } \\
\text { METHANESULFONATE_CTD_00006307 }\end{array}$ & 940 & 3865 & 0.047 \\
\hline
\end{tabular}



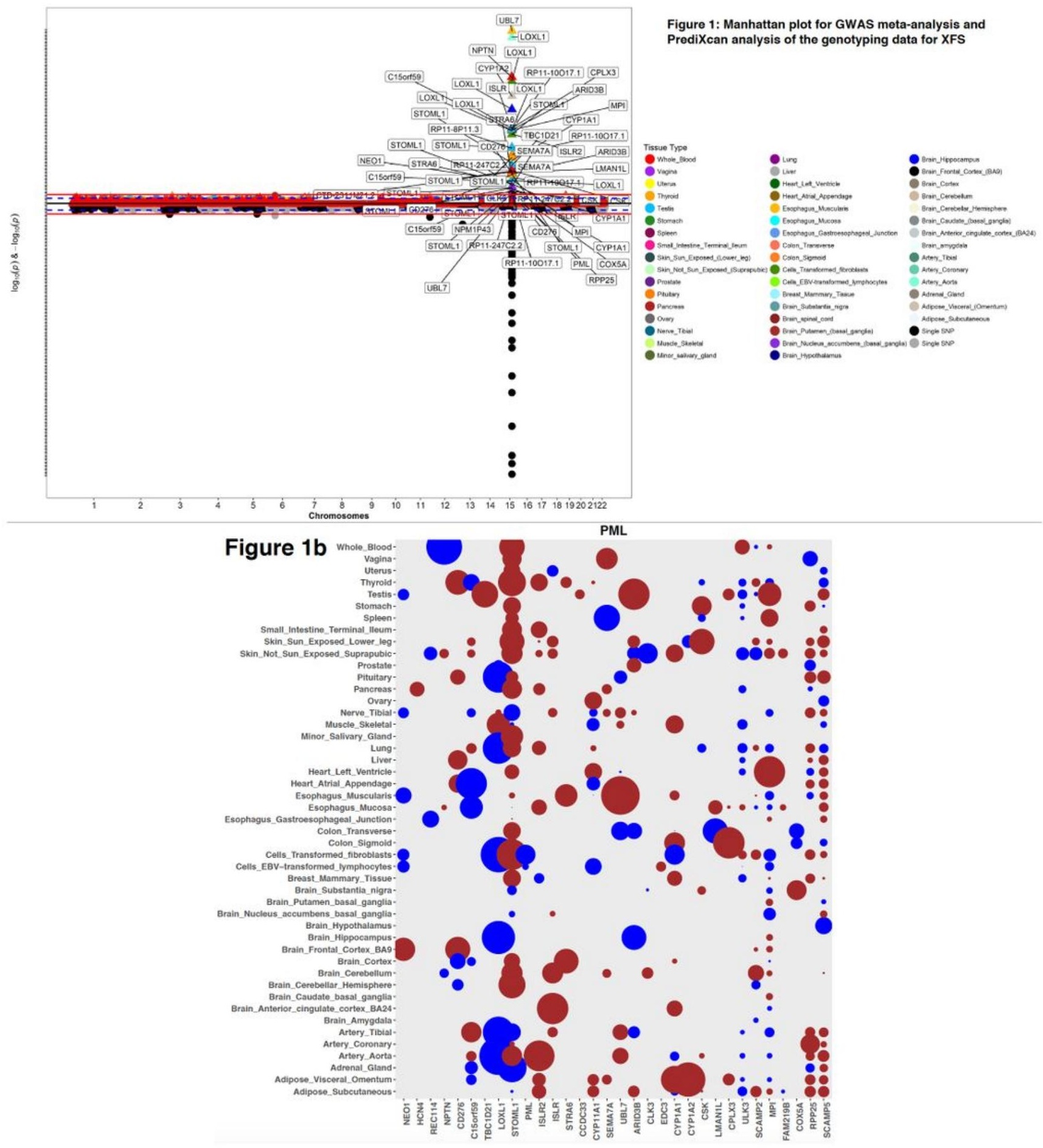

Figure 1

Manhattan plot for GWAS meta-analysis and PrediXcan analysis of the genotyping data for XFS. a) The lower half of the plot is for the XFS meta-analysis summary statistics data Aung et al., 2017, while the upper half of the plot shows results from PrediXcan analysis for 48 GTEx tissues. On the X axis is plot of 
variant/gene associations along the chromosomes, while $Y$ axis represent the significance levels for the associations. The legend for PrediXcan analysis on the 48 GTEx tissues, a color for each tissue, is on the right. For both plots the blue dotted line is the "suggestive" genome-wide significant threshold $(p<1 e-4)$, while the red line is the genome-wide significant threshold. On the lower plot, the gene labels are for genes reported/mapped to genome-wide significant signals in GWAS result, while in the upper plot is for genes that are associated at "suggestive" significant threshold. b) genes in the region in chromosome 15 that show significant association. P values for the plot in Suppl. Table S2. C15orf59 is INSYN1.

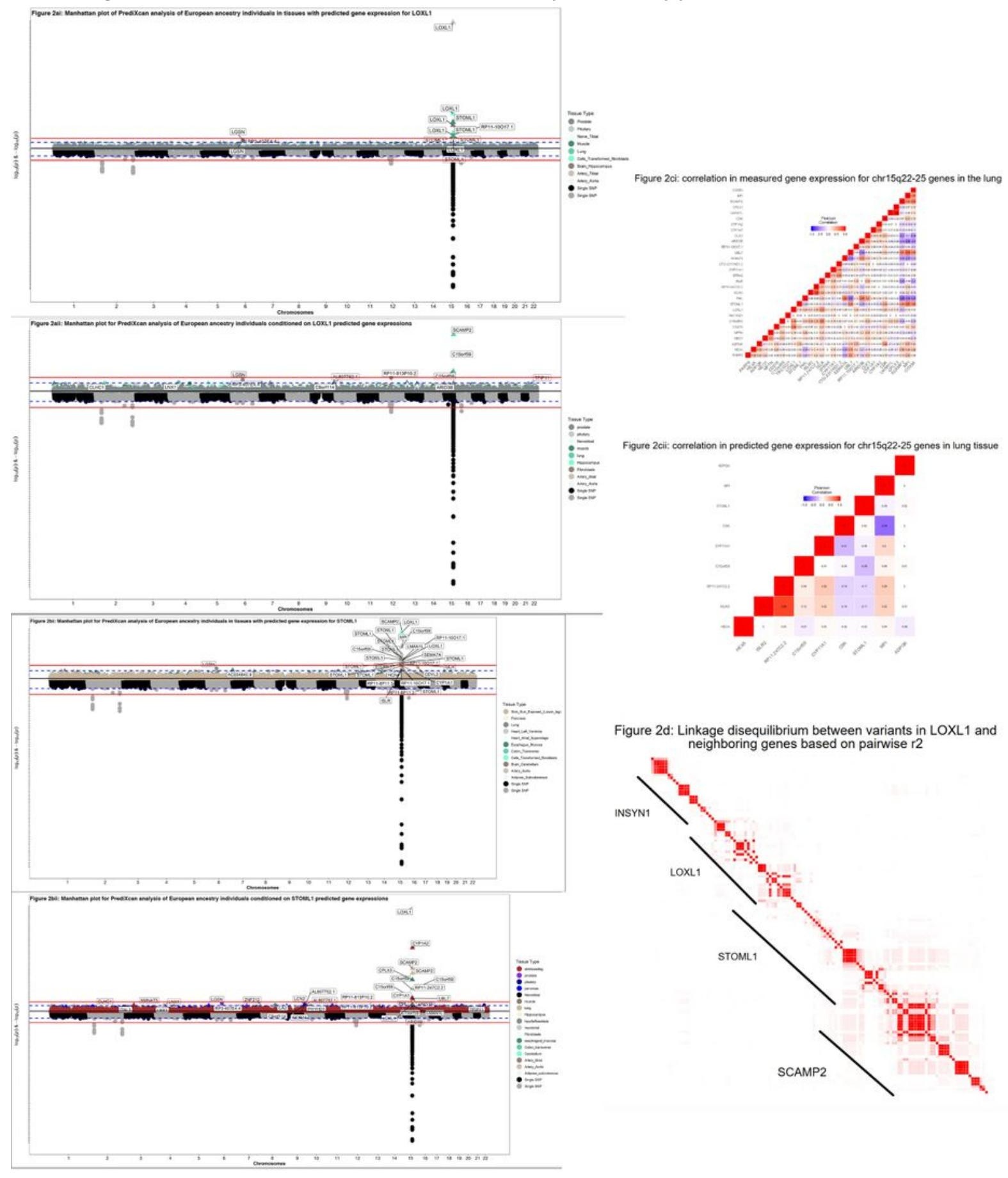


Figure 2

Conditional analysis to prioritize XFS associated genes: a) Manhattan plot for PrediXcan analysis of European ancestry individuals in tissues with predicted gene expression for a) i) LOXL1 and ii) conditioned on LOXL1 predicted gene expressions b) Manhattan plot for PrediXcan analysis of European ancestry individuals in tissues with predicted gene expression for i) STOML1 and ii) conditioned on STOML1 predicted gene expressions c) correlation in gene expression in for genes in chr15q22-25 in lung tissue for i) reference GTEx data ii) predicted gene expression in BioVU cohort. In each case on the $\mathrm{X}$ axis is plot of variant/gene associations along the chromosomes, while $Y$ axis represent the significance levels for the associations. The legend for PrediXcan analysis on the GTEx tissues, a color for each tissue, is on the right. For both plots the blue dotted line is the "suggestive" genome-wide significant threshold $(p<1 e-4)$, while the red line is the genome-wide significant threshold. On the lower plot, the gene labels are for genes reported/mapped to genome-wide significant signals in GWAS result, while in the upper plot is for genes that are associated at "suggestive" significant threshold. P values for the plot in Suppl. Table S2. d) linkage disequilibrium between variants in prediction models for LOXL1 and neighboring genes in lung tissue based on pairwise r2. C15orf59 is INSYN1.

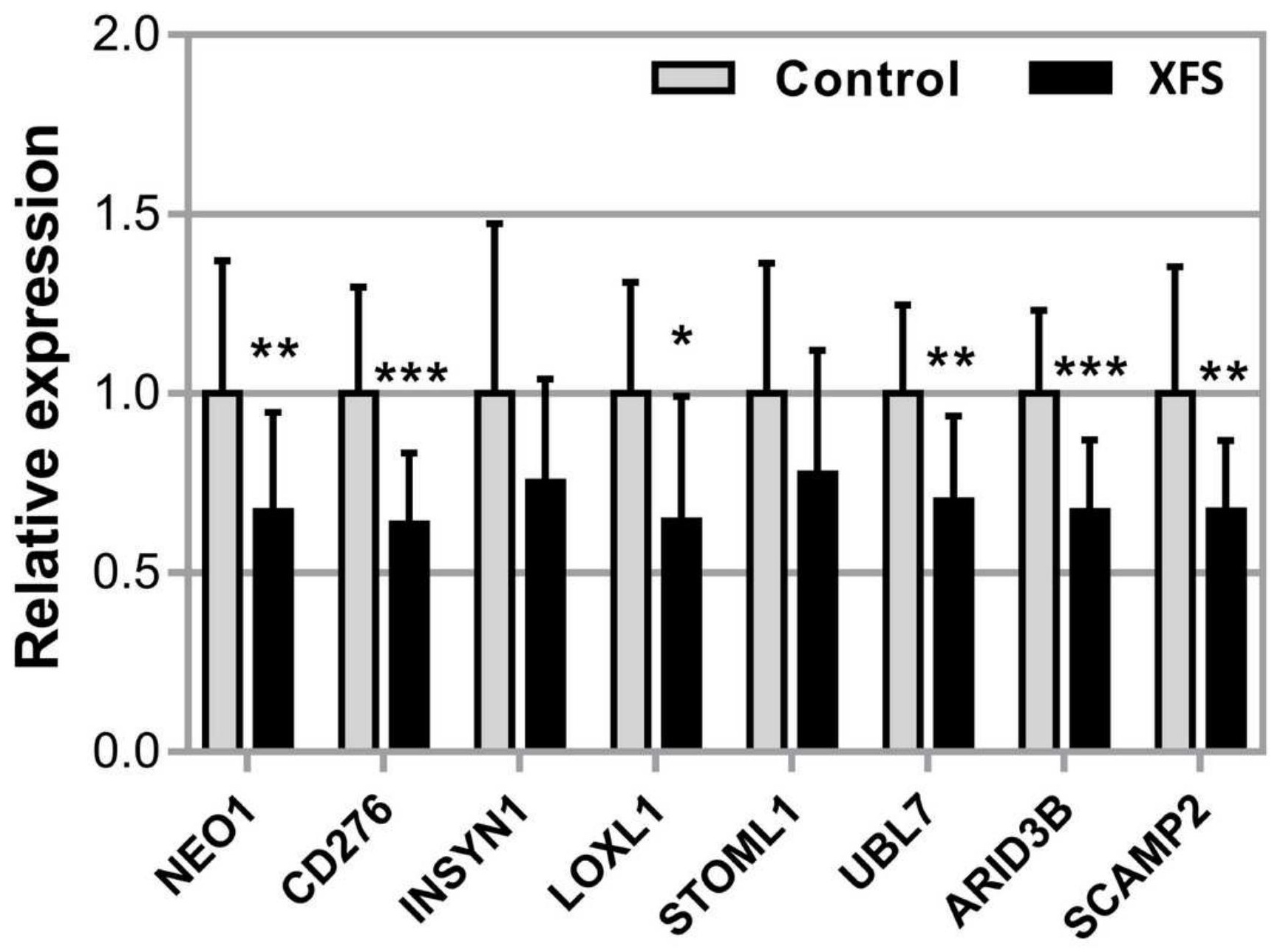

Figure 3 
Expression of NE01, CD276, INSYN1, LOXL1, STOML1, UBL7, ARID3B and SCAMP2 mRNA in iris tissues derived from normal human donors (control) $(n=19)$ and donors with XFS syndrome $(n=12)$ using realtime PCR technology. Expression levels were reduced in XFS specimens compared to control specimens, with significant differences for NE01, CD276, LOXL1, UBL7, ARID3B and SCAMP2. The relative expression levels were normalized relative to GAPDH and are represented as mean values $\pm S D\left({ }^{\star} p<0.05 ;{ }^{* \star} p<0.01\right.$, $\star \star \star p<0.001)$.

\section{Supplementary Files}

This is a list of supplementary files associated with this preprint. Click to download.

- SupplementaryInformationandFigures.pdf

- Suppl.TablesS26PrediXcanResults.xIsx

- Suppl.TablesS7PrediXcaninreducedmodels.xlsx

- Suppl.TablesS8enrichmentanalysis.xIsx

- Suppl.TablesS9PheWASfinal.xlsx 